\title{
Characterization of pectin extracted from Citrus reticulata L. Blanco collected from different altitudes of Sikkim Himalaya
}

\author{
Anjana Pradhan \\ Sikkim University, Department of Horticulture, $6^{\text {th }}$ mile, Tadong (Sikkim), India \\ Laxuman Sharma* \\ Sikkim University, Department of Horticulture, $6^{\text {th }}$ mile, Tadong, (Sikkim), India \\ Archana Tiwari \\ Sikkim University, Department of Physics, Tadong, (Sikkim), India \\ Prajwal Chettri \\ Sikkim University, Department of Physics, Tadong, (Sikkim), India
}

*Corresponding author. E-mail:laxumans@gmail.com

\begin{abstract}
Sikkim mandarin (Citrus reticulata) is most important cash crops of Sikkim Himalaya, a tiny state in North East India. The fruit is usually peeled off and eaten as desert used for extraction of juiceor processed for other products. The peel is thrown as waste, though it is rich commercially important essentialoil andpectin. The pectin can be obtained from the pulp waste after extraction of essential oil. The essential oil and pectin content is the effect of the climatic functions. In Himalayas, there is abrupt change in microclimate with change in the altitude. C. reticulata in Sikkim Himalayas grows at the altitudinal range of 800 to 1800 metre from mean sea level. During the present studies pectin was extracted from peel waste after extraction of essential oil. The fruits were collected from five different altitude range viz: $800-1000 \mathrm{~m}, 1000-1200 \mathrm{~m}, 1200-1400 \mathrm{~m}, 1400-1600 \mathrm{~m}$ and $>1600 \mathrm{~m}$. FTIR works on the basis of functional group showed range from $3607 \mathrm{~cm}^{-1}(\mathrm{O}-\mathrm{H}$ stretch region) to $748 \mathrm{~cm}^{-1}$ (C-H bend) in mature stage and $3585 \mathrm{~cm}^{-1}(\mathrm{O}-\mathrm{H}$ stretch) to $883(\mathrm{C}-\mathrm{Cl}$ stretch) $\mathrm{cm}^{-1}$ in immature stage. Moreover essential oil showed different compound identification. Limonene was found to be the highest at $>1600 \mathrm{~m}$ altitude $(88.46 \%)$ at mature stage and $(89.06 \%)$ at immature stage respectively. These variation may be due to different climatic condition and soil of the elevation. The overall results showed that the pectin can be beneficial for industrial use as well as in pharmaceutical health promotion and treatment. Further peel of the species can be evaluated for its rich content of limonene by different industries.
\end{abstract}

Keywords: Altitude, Essential oil, Pectin, Pulp waste, Sikkim mandarin (Citrus reticulata)

\section{INTRODUCTION}

Pectin is a biodegradable and natural gelling agent which can be used for commercial purpose (Chin et al., 2014) such as making jam, jellies and marmalade ( Devi et al., 2014) and in fruit juices and milk drink as a stabilizer and also as dietary fiber (Tobias et al., 2011). Pectin is produced as bi - product after juice extraction from citrus peel or apple pomace. Apple pomace gives $10-15 \%$ pectin and that of citrus fruit peel $20-30 \%$ (Kulkarni et al., 2006). Productionof pectin differs between plant to plant, various parts of the single plant, and in the same plant in different time interval (Krishnamurthi andGiri, 2003). Considering the fact, present studies envisage for extraction of pectin from the citrus (Citrus reticulata)peel after the extraction of essential oil.

\section{Article Info}

DOI:10.31018/jans.v11i1.1997 Received: February 1, 2019

Revised: February 22, 2019

Accepted: February 27, 2019

\section{How to Cite}

Pradhan, A. et al. (2019). Characterization of pectin extracted from Citrus reticulata L. Blanco collected from different altitudes of Sikkim Himalaya. Journal of Applied and Natural Science, 11(1): 168-181 
$\mathrm{pH}$ 2. The mixture was heated at $60^{\circ} \mathrm{C}$ and continuously stirred for 1 hour. The acid extract was then filtered using Whatman No. 1 filter paperand the filtrate was coagulated with an equal volume of $95 \%$ ethanol. The coagulated filtrate waskept undisturbed for 2 hours to make the pectin float on the surfaceasgelatinous jelly. The pectin jelly was filtered and washed with ethyl alcohol 2-3 times to remove impurities. The precipitate was then dried in a hot air oven at $35-40^{\circ} \mathrm{C}$. The yield of pectin (fresh weight anddry weight basis) was calculated in digital weighing balance (Khule et al.,2012).

$$
\text { Ypec }(\%) P \times 100 \quad \text {.......Eq } 1
$$

$B i$

Where, $\mathrm{Y}$ is the yield of pectin in (\%), $\mathrm{P}$ is the amount of extracted pectin in gram and $B$ is the initial weight of fruit peel powder.

Physicochemical characterization of the pectin: The dried pectin samples were subjected to the following qualitative and quantitative tests in order to characterize them.

\section{Qualitative tests}

Color: This was done by visual observation

Solubility of dry pectin in cold and hot water: To determine the solubility of dry pectin, $0.25 \mathrm{~g}$ of pectin powdered sample were placed in two different conical flask and $10 \mathrm{~mL}$ of $95 \%$ ethanol and 50 $\mathrm{mL}$ of distilled water was added. The mixture in one flask was kept and other was shaken vigorously toform a suspension and then heated at 85$95^{\circ} \mathrm{C}$ for 15 min (Fishman et al., 2003).

Solubility of pectin solution in cold and hot alkali (NaOH): In two different conical flasks $1 \mathrm{ml}$ of $0.1 \mathrm{~N} \mathrm{NaOH}$ was placed and $5 \mathrm{ml}$ of pectin solution was added. Further second flask was heated at $85-90^{\circ} \mathrm{C}$ for $15 \mathrm{~min}$ (Joslyn, 1980).

Sugar and organic acids: $1 \mathrm{~g}$ of pectin samplewas placed in $500 \mathrm{~mL}$ flask and5 $\mathrm{mL}$ ethanol and $100 \mathrm{~mL}$ water was poured rapidly, shaken and then allowed to stand for 10 minutes. To this solution, $100 \mathrm{~mL}$ of ethanol containing $0.3 \mathrm{~mL}$ hydrochloric acid was mixed and then filtered using what man No1 filter. The filtrate around $2.5 \mathrm{~mL}$ was measured into a conical flask $(25 \mathrm{~mL})$, the liquid was placed in steam bath for evaporation and residue was dried in a hot air oven at $50^{\circ} \mathrm{C}$ for $2 \mathrm{~h}$ (Devanooru et al., 2015)

\section{Quantitative Tests}

Equivalent weight determination: Equivalent weight was determined from $0.5 \mathrm{~g}$ of pectin sample, to which $5 \mathrm{~mL}$ ethanol was added followed by 1 $\mathrm{g}$ sodium chloride and $100 \mathrm{~mL}$ of distilled and few drops of phenol red indicator. The mixture was shaken well to avoid the clumping and titrated against0.1 $\mathrm{M} \mathrm{NaoH}$ to a pink colour at the endpoint (Owens et al., 1952).

Following equation was used to calculate equivalent weight of pectin. (Krishnamurthi and Giri, 2003).

Equivalent Weight: (weight of pectin sample $x$
Molarity of alkali) / Volume of alkali x100 ......Eq 2 Methoxyl content determination: The neutralized solution left after determining equivalent weight was placed in a conical flask $(250 \mathrm{~mL})$. In that $25 \mathrm{~mL}$ of $0.25 \mathrm{M} \mathrm{NaOH}$ was added. The mixture was stirred thoroughly and allowed to stand for $30 \mathrm{~min}$ at ambient temperature (Norziah, et al., 2000). The methoxyl content was calculated using the equation below( Kulkarni et al., 2006 ).

Methoxyl content \% =Volume of alkali $\mathrm{x}$ weight $/$ Weight of pectin samplex $100 \quad$.. . .Eq.3

Moisture content determination: An empty petri dish was kept in a hot air oven, cooled and then weighed in a digital weighing balance. $5 \mathrm{~g}$ of the pectin sample was then transferred into the empty dish, kept in a hot air oven at $130^{\circ} \mathrm{C}$ for $1 \mathrm{~h}$ thereafter the petri dish was removed, cooled indesiccators and weighed.Moisture content was then calculated using Joye and Luzio, 2000

Moisture Content $(\%)=$ Weight of the Residue $/$ Weight of the Sample $x 100 \% \quad$........Eq.4

Anhydrouronic Acid (AUA) Content: Anhydrounic acid content (AUA) was calculated by using values from equivalent weight and methoxyl content. AUA content was then estimated as per (Pagan et al., 2000) (Owens et al., 1952)

AUA\% $=176 / \mathrm{Z}$ Weight of sample $(\mathrm{mg})$ $\mathrm{x} 100 \quad$.............Eq.5

(where, 176 is the molecular weight of AUA) and meq of Titration $A+$ meq of Titration $B$

Degree of esterification (DE): DE \%was calculated by following equation (Khule et al.,2012), using the values from methoxyl and anhydrouronic acid content determinations (Schultz, 1976).

$\mathrm{DE} \%=176 \times \mathrm{MeO} \% \times 100 / 31 \times$ AUA \% ...Eq.6 Ash percentage: An empty crucible was weighed in which $1 \mathrm{~g}$ of pectin was placed and then kept in muffle furnace at $600^{\circ} \mathrm{C}$ for $3-4$ hours. The crucible was then cooled at room temperature in a desiccators and weigh (Ranganna, 1986)

Ash $\%$ was calculated using following equation Ash $\%=$ Weight of ash $\times$ Weight of pectin $\times 100$ ...Eq. 7

Alkalinity percent as carbonate: In $25 \mathrm{ml}$ of 0.1 $\mathrm{N} \mathrm{HCl}$, ash was dissolved. It was heated to boiling and cool. Then titration was done with $0.1 \mathrm{~N}$ $\mathrm{NaOH}$ using phenolphthalein indicators. (Ranganna, 1986)

Alkalinity $\%$ as carbonate $=$ Titre $X$ Normality of $\mathrm{NaOH} /$ Weight of ash X $1000 \times 60 \times 100 \ldots .$. Eq.8

Carbonate free ash \%: It is calculated by using the following equations (Ranganna, 1986) as:

Carbonate free ash \% $=$ Ash $\%$ - Carbonate free ash\% ......Eq.9

Spectral analysis: Subsequent to the above mentioned tests, the pectin from C. limon was further subjected to FTIR analysis (Shimadzu, IRAffinity-1) and the resultingspectrum was studied in order to understand the functional groups present (Kanmani et al., 2014 
Essential oil extraction: Fresh fruits were washed to remove dirt andrind was separated. $500 \mathrm{~g}$ of peelwas subjected to hydro distillation by adding water enough to dissolve the rind in a $2000 \mathrm{ml}$ round bottom flask fitted to a Clevenger apparatus. After 3 hours, oil was collected and stored in brown bottle and stored at $4{ }^{\circ} \mathrm{C}$ until analysis. Separation of oil with water was done using separating funnel, further water was removed by addition of anhydrous sodiumsulphate. All the extracted oil from Citrus germplasm was then subjected for profiling and characterization following standard procedure and protocol as follows.

Profiling of essential oil: Profiling of essential oil was done by GC/MS-QP-2010 plus Ultra (Shimadzu company) using an Rtx-5 MS fused silica capillary column $(30 \mathrm{~m} \times 0.25 \mathrm{~m}$ i.d., film thickness $0.25 \mu \mathrm{m})$.

Gas chromatography-mass spectrometry analysis: A sample of $1 \mu$ l was used in split plot ratio of 100:1. An electron ionization system with ionization energy of $70 \mathrm{eV}$ was used. Helium was used as carrier gas at a flow rate of $1.5 \mathrm{ml} / \mathrm{min}$. Mass scanning range was varied over $50-550 \mathrm{~m} / \mathrm{z}$ while injector and MS transfer line temperatures was set at 220 and $290^{\circ} \mathrm{C}$ respectively.

Compounds identification: Identification of essential oilvolatile compounds was based on the calculation of theirretention indices $(\mathrm{RI})$ relative to $\left(\mathrm{C}_{8}-\mathrm{C}_{22}\right) \mathrm{n}$-alkanes withthose of authentic compounds available in our laboratory. Further identification was made by matching their recordedmass spectra with those stored in the Wiley/NBS massspectral library of the GC-MS data systems and other published mass spectra (Adams, 2001).

\section{RESULTS AND DISCUSSION}

Physico chemical characterization: The physico chemical characterization of dried pectin from peel is presented in Table 2. The degree of esterification was found to be highest in the samples from the altitude $>1600 \mathrm{~m}$ in both mature $(90.94 \%)$ and immature stages $(91.79 \%)$. The data presented in the finding is higher than reported by Fakayode and Abobi (2018) with 60.4\% in Nigeria at higher altitude.

Higher DE i.e. $>50 \%$ is marked as high methoxyl pectin (HMP) which help pectin to form gel quickly at high temperature with effective action on the lipid profile (Brouns et al. 2012; Dominiak et al.2014).

Likewise, equivalent weight was 496.33and 444.49 , respectively for rind taken from mature and immature fruits at $>1600 \mathrm{~m}$. The data in the finding was comparatively lower than reported by Fakayode and Abobi, 2018 who mentioned 599.74 in Nigeria as the value in their result. As mentioned by Yadav et al. (2017), high equivalent weight showed higher level of gel forming ability

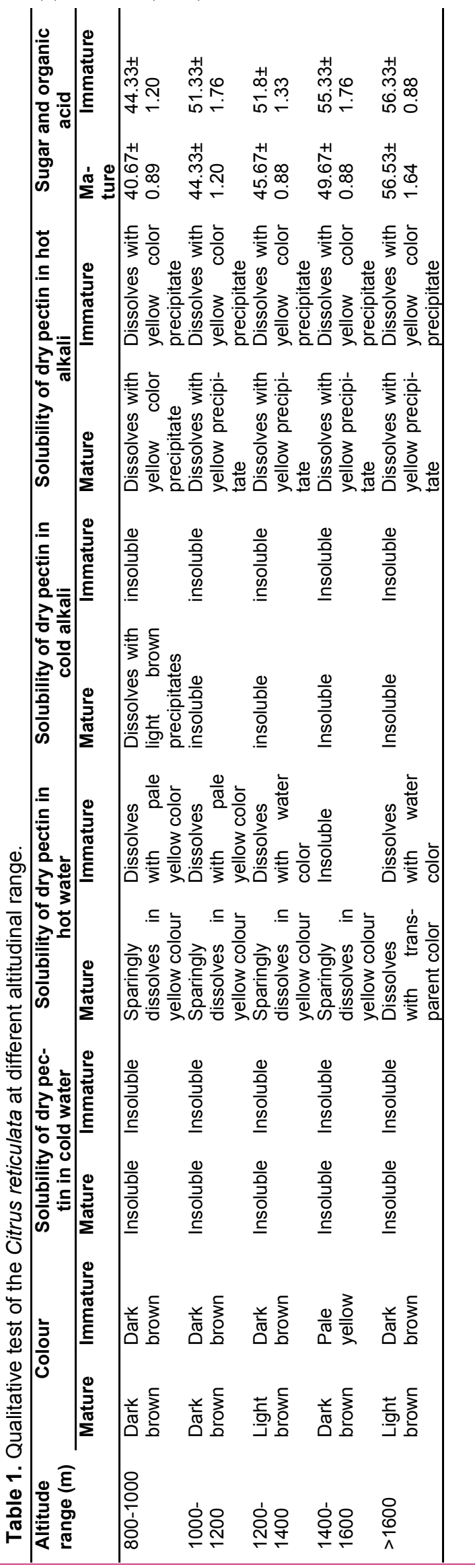


Pradhan, A. et al. / J. Appl. \& Nat. Sci. 11(1): 168-181 (2019)

Table 2. Quantitative tests of Citrus reticulata at different altitudinal range.

\begin{tabular}{lllllll}
\hline Parameters & \multirow{2}{*}{ Stages } & \multicolumn{5}{c}{ Altitude range (m) } \\
\cline { 2 - 6 } & & $\mathbf{8 0 0 - 1 0 0 0}$ & $\mathbf{1 0 0 0 - 1 2 0 0}$ & $\mathbf{1 2 0 0 - 1 4 0 0}$ & $\mathbf{1 4 0 0 - 1 6 0 0}$ & $>\mathbf{1 6 0 0}$ \\
\hline \% yield on dry & Mature & $0.68 \pm 0.43$ & $0.69 \pm 0.22$ & $0.83 \pm 0.17$ & $0.83 \pm 0.13$ & $0.88 \pm 0.30$ \\
basis & Immature & $0.53 \pm 0.31$ & $0.58 \pm 0.32$ & $1.46 \pm 0.14$ & $1.50 \pm 0.15$ & $1.53 \pm 0.03$ \\
$\%$ yield on wet & Mature & $29.49 \pm 1.05$ & $35.83 \pm 1.40$ & $36.82 \pm 1.35$ & $37.00 \pm 0.57$ & $40.82 \pm 0.74$ \\
basis & Immature & $10.00 \pm 0.58$ & $13.25 \pm 0.67$ & $15.21 \pm 0.94$ & $15.10 \pm 0.10$ & $15.21 \pm 0.32$ \\
Equivalent weight & Mature & $395.00 \pm 2.89$ & $412.89 \pm 1.97$ & $451.81 \pm 2.82$ & $494.70 \pm 2.03$ & $496.33 \pm 1.86$ \\
& Immature & $378.82 \pm 1.64$ & $396 \pm 2.31$ & $396.33 \pm 1.86$ & $414.89 \pm 1.06$ & $444.49 \pm 2.79$ \\
Methoxyl Content & Mature & $5.07 \pm 0.07$ & $6.29 \pm 0.04$ & $6.57 \pm 0.05$ & $6.64 \pm 0.15$ & $6.93 \pm 0.03$ \\
(MeO \%) & Immature & $4.32 \pm 0.01$ & $5.33 \pm 0.35$ & $6.24 \pm 0.46$ & $6.46 \pm 0.21$ & $6.91 \pm 0.04$ \\
Anhydrouronic & Mature & $41.95 \pm 0.45$ & $42.03 \pm 0.07$ & $42.58 \pm 0.22$ & $43.20 \pm 0.73$ & $43.27 \pm 0.42$ \\
Acid (AUA \%) & Immature & $37.79 \pm 0.21$ & $41.43 \pm 0.98$ & $41.86 \pm 0.95$ & $42.31 \pm 0.05$ & $43.59 \pm 0.22$ \\
Degree of esterifi- & Mature & $88.23 \pm 0.87$ & $88.65 \pm 0.69$ & $89.23 \pm 0.66$ & $90.75 \pm 0.57$ & $90.94 \pm 0.36$ \\
cation (DE \%) & Immature & $85.67 \pm 0.33$ & $88.90 \pm 0.27$ & $89.33 \pm 0.34$ & $90.34 \pm 0.63$ & $91.79 \pm 0.79$ \\
Moisture & Mature & $56.83 \pm 0.44$ & $63.33 \pm 0.33$ & $64.40 \pm 0.30$ & $65.40 \pm 0.30$ & $66.47 \pm 0.29$ \\
& Immature & $55.00 \pm 0.58$ & $64.00 \pm 0.58$ & $64.33 \pm 0.33$ & $64.96 \pm 0.50$ & $66.3 \pm 0.15$ \\
Ash \% & Mature & $55.00 \pm 2.52$ & $56.57 \pm 2.09$ & $56.74 \pm 2.80$ & $62.33 \pm 1.86$ & $67.53 \pm 1.29$ \\
Alkalinity as car- & Mature & $4.28 \pm 0.09$ & $5.60 \pm 0.60$ & $5.53 \pm 0.76$ & $6.47 \pm 0.20$ & $6.11 \pm 0.17$ \\
bonate & Immature & $4.29 \pm 0.01$ & $6.06 \pm 0.60$ & $6.66 \pm 0.01$ & $7.10 \pm 0.48$ & $7.92 \pm 0.40$ \\
Carbonate free & Mature & $50.72 \pm 2.48$ & $50.98 \pm 2.26$ & $52.05 \pm 2.53$ & $55.87 \pm 1.88$ & $61.42 \pm 1.16$ \\
ash & Immature & $40.04 \pm 0.32$ & $40.34 \pm 0.86$ & $47.34 \pm 0.56$ & $48.90 \pm 0.98$ & $64.41 \pm 1.15$ \\
\hline
\end{tabular}

Table 3a. Functional groups present at mature stage in Sikkim mandarin (Citrusreticulata) at $800 \mathrm{~m}$ altitude.

\begin{tabular}{lll}
\hline Frequency & Bond & Functional group \\
\hline $3607(\mathrm{~m}, \mathrm{~s})$ & O-H stretch & Alcohol \\
$3029(\mathrm{w}, \mathrm{b})$ & C-H stretch & Aromatics \\
$2956(\mathrm{~m})$ & C-H stretch & Alkane \\
$1727(\mathrm{~s})$ & C= O stretch & a, $\beta$ unsaturated ester \\
$1201(\mathrm{~m})$ & C-N stretch & Aliphatic amines \\
$1075(\mathrm{~m})$ & C-N stretch & Aliphatic amines \\
$984(\mathrm{~s})$ & C-H out of & Aromatic \\
& plane blend \\
$867(\mathrm{~s})$ & C-H out of & Aromatic \\
& plane blend \\
\hline
\end{tabular}

Table 3b. Functional groups presentat mature stage in Sikkim mandarin at 1000-1200m altitude.

\begin{tabular}{|c|c|c|}
\hline $\begin{array}{l}\text { Frequency } \\
\text { (cm) }\end{array}$ & Bond & Functional group \\
\hline $3135(w)$ & $\mathrm{O}-\mathrm{H}$ stretch & \multirow{7}{*}{$\begin{array}{l}\text { Alcohol } \\
\text { Alkane } \\
\text { Aldehyde } \\
\text { a, } \beta \text { unsaturated } \\
\text { ketone } \\
\text { Aliphatic amine } \\
\text { Aliphatic amines } \\
\text { Alkene }\end{array}$} \\
\hline $2939(\mathrm{~m})$ & $\mathrm{C}-\mathrm{H}$ stretch & \\
\hline $1721(\mathrm{~s})$ & $\mathrm{C}=\mathrm{O}$ stretch & \\
\hline $1625(\mathrm{~s})$ & $\mathrm{C}=\mathrm{C}$ stretch & \\
\hline $1219(\mathrm{~m})$ & C-N stretch & \\
\hline $1073(\mathrm{~m})$ & C-N stretch & \\
\hline 1002(s) & $\begin{array}{l}\text { C-Hout of } \\
\text { plane blend }\end{array}$ & \\
\hline
\end{tabular}

Table 3c. Functional groups present at mature stage in Sikkim mandarin at 1200-1400m altitude.

\begin{tabular}{|c|c|c|}
\hline $\begin{array}{l}\text { Frequency } \\
\text { (cm) }\end{array}$ & Bond & Functional group \\
\hline $3537(\mathrm{~s})$ & $\begin{array}{l}\mathrm{O}-\mathrm{H} \text { stretch, } \\
\mathrm{H} \text {-bonded }\end{array}$ & Alcohols, phenols \\
\hline $\begin{array}{l}3066(\mathrm{~s}) \\
2957(\mathrm{~m}) \\
1721(\mathrm{~s}) \\
1214(\mathrm{~m}) \\
1074(\mathrm{~m}) \\
1005(\mathrm{~s})\end{array}$ & $\begin{array}{l}\mathrm{C}-\mathrm{H} \text { stretch } \\
\mathrm{C}-\mathrm{H} \text { stretch } \\
\mathrm{C}=\mathrm{O} \text { stretch } \\
\mathrm{C}-\mathrm{N} \text { stretch } \\
\mathrm{C}-\mathrm{N} \text { stretch } \\
\mathrm{C}-\mathrm{H} \text { out of } \\
\text { plane blend }\end{array}$ & $\begin{array}{l}\text { Aromatics } \\
\text { Alkanes } \\
\alpha, \beta \text { unsaturated ester } \\
\text { Aliphatic amines } \\
\text { Aliphatic amines } \\
\text { Alkenes }\end{array}$ \\
\hline
\end{tabular}

Table 3d. Functional groups presentat mature stage in Sikkim mandarin at $1400-1600 \mathrm{~m}$ altitude.

\begin{tabular}{lll}
\hline $\begin{array}{l}\text { Frequency } \\
\text { (cm ) }\end{array}$ & Bond & Functional group \\
\hline $3048(\mathrm{~s})$ & $\mathrm{C}-\mathrm{H}$ & Aromatics \\
$1712(\mathrm{~s})$ & $\mathrm{C}=\mathrm{O}$ & a, $\beta$ unsaturated ester \\
$1619(\mathrm{~m}-\mathrm{w})$ & $\mathrm{C}=\mathrm{C}$ & Alkenes \\
$1209(\mathrm{vs})$ & $\mathrm{C}-\mathrm{N}$ stretch & Aliphatic amines \\
$1103(\mathrm{~s})$ & $\mathrm{C}-\mathrm{H}$ wag & Alkyl halides \\
& $\left(-\mathrm{CH}_{2} \mathrm{X}\right)$ & \\
$1064(\mathrm{~m})$ & $\mathrm{C}-\mathrm{N}$ stretch & Aliphatic amines \\
$1017(\mathrm{~m})$ & $\mathrm{C}-\mathrm{N}$ stretch & Aliphatic amines \\
$748(\mathrm{~s})$ & $\mathrm{C}-\mathrm{Hout}$ of & Alkene \\
& plane blend & \\
\hline
\end{tabular}

Table 3e. Functional groups present at mature stagein Sikkim mandarinat $>1600 \mathrm{~m}$.

\begin{tabular}{|c|c|c|}
\hline $\begin{array}{l}\text { Frequency } \\
(\mathrm{cm})\end{array}$ & Bond & $\begin{array}{l}\text { Functional } \\
\text { group }\end{array}$ \\
\hline 3062 (s) & C-H stretch & Aromatics \\
\hline 2959 (m) & C-H stretch & Alkane \\
\hline 1715 (s) & $\mathrm{C}=\mathrm{O}$ stretch & $\begin{array}{l}\alpha, \beta \text { unsaturated } \\
\text { ester }\end{array}$ \\
\hline $1613(m-w)$ & $\mathrm{C}=\mathrm{C}$ stretch & Alkene \\
\hline 1214 (s) & C-N stretch & Aliphatic amine \\
\hline $1108(\mathrm{~m})$ & $\mathrm{C}-\mathrm{H}$ wag $\left(-\mathrm{CH}_{2} \mathrm{X}\right)$ & Alkyl halides \\
\hline $1095(\mathrm{~m})$ & C-N stretch & Aliphatic amine \\
\hline 1021 (s) & C-N stretch & Aliphatic amine \\
\hline
\end{tabular}

Table 3f. Functional groups present at immature stage in Sikkim mandarin (Citrus reticulata) at $800 \mathrm{~m}$ altitude.

\begin{tabular}{lll}
\hline $\begin{array}{l}\text { Frequency } \\
\text { (cm )48 }\end{array}$ & Bond & Functional group \\
\hline $3364(\mathrm{~s})$ & O-H stretch & Alcohol \\
$2949(\mathrm{~m})$ & C-H stretch & Alkane \\
$1722(\mathrm{~s})$ & C=O stretch & $\alpha, \beta$ unsaturated ester \\
$1204(\mathrm{v}, \mathrm{s})$ & C-O stretch & Phenols \\
$1026(\mathrm{~m}-\mathrm{s})$ & C-N stretch & Amines \\
\hline
\end{tabular}


Table $\mathbf{3 g}$. Functional groups present at immature stage in Sikkim mandarin (Citrus reticulata) at 1000 $-1200 \mathrm{~m}$ altitude.

\begin{tabular}{lll}
\hline $\begin{array}{l}\text { Frequency } \\
\text { (cm ) }\end{array}$ & Bond & Functional group \\
\hline $3514(\mathrm{~s}, \mathrm{sh})$ & $\begin{array}{l}\text { O-H stretch, } \\
\text { H-bonded }\end{array}$ & Alcohols, phenols \\
& O-H bend & Carboxylic acid \\
3096(m) & C-H stretch & Alkanes \\
$2993(\mathrm{~s})$ & C=O stretch & Carboxylic acid \\
$1692(\mathrm{~s})$ & C-N stretch & Aliphatic amines \\
$1209(\mathrm{~s})$ & C-N stretch & Aliphatic amines \\
$1096(\mathrm{~m})$ & C-N stretch & Aliphatic amines \\
\hline
\end{tabular}

Table $3 \mathrm{~h}$. Functional groups present at immature stage in Sikkim mandarin (Citrus reticulata) at 1200 $-1400 \mathrm{~m}$ altitude.

\begin{tabular}{lll}
\hline $\begin{array}{l}\text { Frequency } \\
\text { (cm ) }\end{array}$ & Bond & Functional group \\
\hline $3585(\mathrm{~s})$ & O-H stretch & $\begin{array}{l}\text { Alcohols, phenols, H- } \\
\text { bonded }\end{array}$ \\
$3048(\mathrm{~s})$ & C-H stretch & Alkane \\
$2932(\mathrm{~m})$ & C-H stretch & Alkane \\
$1714(\mathrm{~s})$ & C=O stretch & $\alpha, \beta$ unsaturated ester \\
$1229(\mathrm{~s})$ & C-N stretch & Aliphatic amines \\
$1075(\mathrm{~m})$ & C-N stretch & Aliphatic amines \\
$1018(\mathrm{~m})$ & C-N stretch & Aliphatic amines \\
\hline
\end{tabular}

while at lower levelthere will be higher partial degradation of the pectin which is disadvantageous.

On the other hand, respective $\mathrm{MeO}$ was 6.93and 6.91 in mature and immature stage at $>1600 \mathrm{~m}$ altitude. The results were in corroboration with Fakayode and Abobi, 2018, who reported6.23\% $\mathrm{MeO}$ in Nigeria at higher altitudein orange peel. In 2014, Kanmani et al., illustrated MeO range from $0-12 \%$ based on source and method of extraction. $\mathrm{MeO}$ less than $7 \%$, is considered to be the pectin of good quality as mentioned by (Bagde et al., 2017) who had done research in Yavatmal. Low $\mathrm{MeO}$ contributes furthermore-irreversible gel properties, which means it will remain in gelled form even if heated at melting temperature. Further, they can be used in making low sugar jam in food industry as itdoes not require high sugar for forming gel They can also be used in various aspects as gelling agent, thickening agent and fat substitute (Tiwari et al. 2017).

The total anhydrouronic acid content was found to be $43.27 \%$ and $43.59 \%$ at $>1600 \mathrm{~m}$ altitude while the lowest was accorded in $800 \mathrm{~m}$ with $41.95 \%$ and $37.79 \%$ in mature and immature stages respectively. Low value of AUA means that the extracted pectin might have high amount of protein, starch and sugars in the precipitated pectin in Bangladesh (Ismail et al., 2012).

Moisture content of the pectin was also estimatedhighin $>1600 \mathrm{~m}$ altitude with $66.70 \%$ and $64.30 \%$, respectively. It was higher than orange peel $(50 \%)$ and lower than lemon peels $(70 \%)$ as reported by Bagde et al., 2017in Yavatmal.

Yield of pectin on wet basis showed $40.82 \%$,
Table 3i. Functional groups present at immature stage in Sikkim mandarin (Citrus reticulata) at 1400-1600m altitude.

\begin{tabular}{lll}
\hline $\begin{array}{l}\text { Frequency } \\
\text { (cm ) 5 }\end{array}$ & Bond & Functional group \\
\hline $2952(\mathrm{~m})$ & C-H stretch & Alkane \\
$2617(\mathrm{~m})$ & O-H stretch & Carboxylic acid \\
$1990(\mathrm{~m})$ & C-N stretch & Aliphatic amines \\
$1717(\mathrm{~s})$ & C=O stretch & $\alpha, \beta$ unsaturated ester \\
$1620(\mathrm{~s})$ & C=C stretch & Vinyl ether \\
$1339(\mathrm{~m}, \mathrm{w})$ & C-N stretch & Amine \\
$1206(\mathrm{~s})$ & C-N stretch & Aliphatic amines \\
$1074(\mathrm{~m})$ & C-N stretch & Aliphatic amines \\
$883(\mathrm{~m})$ & C-Cl stretch & Alkyl halides \\
\hline
\end{tabular}

Table 3j. Functional groups present at immature stage in Sikkim mandarin (Citrus reticulata) at $>1600 \mathrm{~m}$ altitude.

\begin{tabular}{|c|c|c|}
\hline $\begin{array}{l}\text { Frequency } \\
\text { (cm )44 }\end{array}$ & Bond & Functional group \\
\hline $3604(\mathrm{~s})$ & $\mathrm{O}-\mathrm{H}$ stretch & Alcohol, phenols \\
\hline 3042(b) & $\mathrm{O}-\mathrm{H}$ stretch & Carboxylic acid \\
\hline $2957(\mathrm{~m}-\mathrm{s})$ & $\mathrm{C}-\mathrm{H}$ stretch & Alkyl \\
\hline 1696(s) & $\mathrm{C}=\mathrm{O}$ stretch & $\begin{array}{l}\text { Unsaturated/aromatic } \\
\text { carboxylic acid }\end{array}$ \\
\hline $1210(s)$ & C-O stretch & Ether \\
\hline $\begin{array}{l}\text { 1075(often } \\
\text { over lapped) }\end{array}$ & C-N stretch & Aliphatic amines \\
\hline $\begin{array}{l}\text { 1019(often } \\
\text { over lapped) }\end{array}$ & C-N stretch & Aliphatic amines \\
\hline
\end{tabular}

Note : $s=$ strong,$m=$ medium, $w=$ weak,$b=$ bend

$15.21 \%$ in mature and immature stages while $0.88 \%$ and $1.5 \%$ in mature and immature stages on dry basis respectively. The data in the present findings was comparatively higher for wet and dry basis as illustrated by Bagde et al., 2017 in lemon peel and orange peel are $11,8 \%$ while on dry basis were $1,0.5 \%$, respectively.

The data regarding ash $\%$ showed $72.33 \%$ and $67.53 \%$ occupying the maximum value at $>1600 \mathrm{~m}$ altitude in mature and immature stages which contradicts the finding of Bagde et al., 2017 wherein, only $30 \%$ and $35 \%$ of ash content in lemon and orange peel pectin was reported. Likewise alkalinity as carbonate and carbonate free ash were studied which revealed that immature $(6.92,64.41)$ has high value compared to mature stage $(6.11$, 61.42 ) and was found to be higher at $>1600 \mathrm{~m}$ altitudes.

There is considerable variation in physic chemical characterization of pectin from different altitude.

It may be due to variability in soil characteristics and climatic conditions (rainfall, temperature and humidity) in the various altitudes under research. It can also be revealed that geographical location plays a major role in the alteration of these parameters.

FTIR Spectral analysis: The FTIR spectrum of pectin extracted from Sikkim mandarin is illustrated in Fig.1 and corresponding functional groups 
Pradhan, A. et al. / J. Appl. \& Nat. Sci. 11(1): 168-181 (2019)

Table 4a. Composition of essential oil compounds at $800 \mathrm{~m}$ altitude in mature stage.

\begin{tabular}{|c|c|c|c|c|c|c|}
\hline S.N. & Compound name & Area \% & $\mathbf{R I}$ & R. Time & $\begin{array}{l}\text { Molecular } \\
\text { formula }\end{array}$ & $\begin{array}{l}\text { Molecular } \\
\text { weight }\end{array}$ \\
\hline 1. & $\alpha$-Thujene & 0.1634 & 922 & 8.449 & $\mathrm{C}_{10} \mathrm{H}_{16}$ & 136 \\
\hline 2. & $\alpha$-Pinene & 0.7776 & 929 & 8.735 & $\mathrm{C}_{10} \mathrm{H}_{16}$ & 136 \\
\hline 3. & Camphene & 0.0044 & 945 & 9.374 & $\mathrm{C}_{10} \mathrm{H}_{16}$ & 136 \\
\hline 4. & Sabinene & 0.5019 & 969 & 10.329 & $\mathrm{C}_{10} \mathrm{H}_{16}$ & 136 \\
\hline 5. & $\beta$-Pinene & 0.5504 & 973 & 10.526 & $\mathrm{C}_{10} \mathrm{H}_{16}$ & 136 \\
\hline 6. & Myrcene & 1.6476 & 988 & 11.068 & $\mathrm{C}_{10} \mathrm{H}_{16}$ & 136 \\
\hline 7. & Octanal & 0.4249 & 1003 & 11.665 & $\mathrm{C}_{8} \mathrm{H}_{16} \mathrm{O}$ & 128 \\
\hline 8. & $(+)$-2-Carene & 0.0643 & 1015 & 12.279 & $\mathrm{C}_{10} \mathrm{H}_{16}$ & 136 \\
\hline 9. & Limonene & 88.3991 & 1042 & 13.243 & $\mathrm{C}_{10} \mathrm{H}_{16}$ & 136 \\
\hline 10. & $\beta$-Ocimene & 0.0448 & 1048 & 13.666 & $\mathrm{C}_{10} \mathrm{H}_{16}$ & 136 \\
\hline 11. & y -Terpinene & 4.6372 & 1061 & 14.249 & $\mathrm{C}_{10} \mathrm{H}_{16}$ & 136 \\
\hline 12. & Acetophenone & 0.0160 & 1064 & 14.511 & $\mathrm{C}_{8} \mathrm{H}_{8} \mathrm{O}$ & 120 \\
\hline 13. & 1-Octanol & 0.0653 & 1071 & 14.825 & $\mathrm{C}_{8} \mathrm{H}_{18} \mathrm{O}$ & 130 \\
\hline 14. & $\alpha$-Terpinolene & 0.1836 & 1084 & 15.414 & $\mathrm{C}_{10} \mathrm{H}_{16}$ & 136 \\
\hline 15. & $\alpha$-p-dimethylstyrene & 0.0077 & 1088 & 15.649 & $\mathrm{C}_{8} \mathrm{H}_{18} \mathrm{O}$ & 130 \\
\hline 16. & Linalool & 1.4607 & 1102 & 16.202 & $\mathrm{C}_{10} \mathrm{H}_{18} \mathrm{O}$ & 154 \\
\hline 17. & Nonanal & 0.0243 & 1104 & 16.340 & $\mathrm{C}_{9} \mathrm{H}_{18} \mathrm{O}$ & 142 \\
\hline 18. & Trans-p-Mentha-2,8-dienol & 0.0136 & 1120 & 17.607 & $\mathrm{C}_{10} \mathrm{H}_{16} \mathrm{O}$ & 152 \\
\hline 19. & Cis-p-Mentha-2,8-dien-1-ol & 0.0121 & 1134 & 17.826 & $\mathrm{C}_{10} \mathrm{H}_{16} \mathrm{O}$ & 152 \\
\hline 20. & Citronellal & 0.0499 & 1150 & 18.537 & $\mathrm{C}_{10} \mathrm{H}_{18} \mathrm{O}$ & 154 \\
\hline 21. & Terpinen-4-ol & 0.1188 & 1178 & 19.893 & $\mathrm{C}_{10} \mathrm{H}_{18} \mathrm{O}$ & 154 \\
\hline 22. & $\alpha$-Terpineol & 0.1518 & 1193 & 20.614 & $\mathrm{C}_{10} \mathrm{H}_{18} \mathrm{O}$ & 154 \\
\hline 23. & Decanal & 0.1630 & 1205 & 21.088 & $\mathrm{C}_{10} \mathrm{H}_{20} \mathrm{O}$ & 156 \\
\hline 24. & Nerol & 0.0148 & 1223 & 21.981 & $\mathrm{C}_{10} \mathrm{H}_{18} \mathrm{O}$ & 154 \\
\hline 25. & Carvacryl methyl ether & 0.0680 & 1228 & 22.174 & $\mathrm{C}_{11} \mathrm{H}_{16} \mathrm{O}$ & 164 \\
\hline 26. & Neral & 0.0281 & 1236 & 22.571 & $\mathrm{C}_{10} \mathrm{H}_{16} \mathrm{O}$ & 152 \\
\hline 27. & Carvone & 0.0093 & 1240 & 22.810 & $\mathrm{C}_{10} \mathrm{H}_{14} \mathrm{O}$ & 150 \\
\hline 28. & Geranial & 0.0310 & 1265 & 23.932 & $\mathrm{C}_{10} \mathrm{H}_{16} \mathrm{O}$ & 152 \\
\hline 29. & Perillaldehyde & 0.0384 & 1271 & 24.244 & $\mathrm{C}_{10} \mathrm{H}_{14} \mathrm{O}$ & 150 \\
\hline 30. & o-Cymen-5-ol & 0.1283 & 1292 & 25.262 & $\mathrm{C}_{10} \mathrm{H}_{14} \mathrm{O}$ & 150 \\
\hline 31. & Undecanal & 0.0096 & 1306 & 25.588 & $\mathrm{C}_{11} \mathrm{H}_{22} \mathrm{O}$ & 170 \\
\hline 32. & a-Elemene & 0.0320 & 1332 & 26.916 & $\mathrm{C}_{15} \mathrm{H}_{24}$ & 204 \\
\hline 33. & $\beta$-Elemene & 0.0168 & 1386 & 29.316 & $\mathrm{C}_{15} \mathrm{H}_{24}$ & 204 \\
\hline 34. & Dodecanal & 0.0200 & 1407 & 30.203 & $\mathrm{C}_{12} \mathrm{H}_{24} \mathrm{O}$ & 184 \\
\hline 35. & Germacrene B & 0.0094 & 1426 & 31.015 & $\mathrm{C}_{15} \mathrm{H}_{24}$ & 204 \\
\hline 36. & $\beta$-Farnesene & 0.0200 & 1451 & 32.017 & $\mathrm{C}_{15} \mathrm{H}_{24}$ & 204 \\
\hline 37. & Germacrene D & 0.0535 & 1476 & 33.157 & $\mathrm{C}_{15} \mathrm{H}_{24}$ & 204 \\
\hline 38. & $\alpha$-Farnesene & 0.0106 & 1502 & 34.154 & $\mathrm{C}_{15} \mathrm{H}_{24}$ & 204 \\
\hline 39. & $\mathrm{y}$-Elemene & 0.0279 & 1553 & 36.288 & $\mathrm{C}_{15} \mathrm{H}_{24}$ & 204 \\
\hline
\end{tabular}

Table 4b. Composition of essential oil compounds at 1000-1200m altitude in mature stage.

\begin{tabular}{|c|c|c|c|c|c|c|}
\hline $\begin{array}{l}\text { S. } \\
\text { N. }\end{array}$ & Compound name & Area \% & $\mathbf{R I}$ & R. Time & $\begin{array}{l}\text { Molecular } \\
\text { formula }\end{array}$ & $\begin{array}{l}\text { Molecular } \\
\text { weight }\end{array}$ \\
\hline 1. & $\alpha$-Thujene & 0.1009 & 937 & 8.450 & $\mathrm{C}_{10} \mathrm{H}_{16}$ & 136 \\
\hline 2. & a -Pinene & 0.5270 & 945 & 8.734 & $\mathrm{C}_{10} \mathrm{H}_{16}$ & 136 \\
\hline 3. & Camphene & 0.0057 & 961 & 9.376 & $\mathrm{C}_{10} \mathrm{H}_{16}$ & 136 \\
\hline 4. & Sabinene & 1.1344 & 986 & 10.332 & $\mathrm{C}_{10} \mathrm{H}_{16}$ & 136 \\
\hline 5. & $\beta$-Pinene oxide & 0.7572 & 991 & 10.527 & $\mathrm{C}_{10} \mathrm{H}_{16} \mathrm{O}$ & 152 \\
\hline 6. & Myrcene & 1.5293 & 1004 & 11.061 & $\mathrm{C}_{10} \mathrm{H}_{16}$ & 136 \\
\hline 7. & Octanal & 0.2853 & 1016 & 11.651 & $\mathrm{C}_{8} \mathrm{H}_{16} \mathrm{O}$ & 128 \\
\hline 8. & $(+)-2$ Carene & 0.0797 & 1030 & 12.267 & $\mathrm{C}_{10} \mathrm{H}_{16}$ & 136 \\
\hline 9. & Limonene & 87.6443 & 1057 & 13.190 & $\mathrm{C}_{10} \mathrm{H}_{16}$ & 136 \\
\hline 10. & $\beta$-Ocimene & 0.1376 & 1063 & 13.652 & $\mathrm{C}_{10} \mathrm{H}_{16}$ & 136 \\
\hline 11. & Y -Terpinene & 4.3163 & 1076 & 14.224 & $\mathrm{C}_{10} \mathrm{H}_{16}$ & 136 \\
\hline 12. & 1-Octanol & 0.2077 & 1087 & 14.829 & $\mathrm{C}_{8} \mathrm{H}_{18} \mathrm{O}$ & 130 \\
\hline 13. & Terpinolene & 0.1891 & 1100 & 15.407 & $\mathrm{C}_{10} \mathrm{H}_{16}$ & 136 \\
\hline 14. & p-Cymenene & 0.0170 & 1104 & 15.637 & $\mathrm{C}_{10} \mathrm{H}_{12}$ & 132 \\
\hline \multirow[t]{2}{*}{15.} & Linalool & 1.9045 & 1117 & 16.200 & $\mathrm{C}_{10} \mathrm{H}_{18} \mathrm{O}$ & 154 \\
\hline & & & & & & Contd........ \\
\hline
\end{tabular}


Pradhan, A. et al. / J. Appl. \& Nat. Sci. 11(1): 168-181 (2019)

\begin{tabular}{|c|c|c|c|c|c|c|}
\hline 16. & Nonanal & 0.0365 & 1120 & 16.330 & $\mathrm{C}_{8} \mathrm{H}_{16} \mathrm{O}$ & 128 \\
\hline 17. & Citronellal & 0.1046 & 1166 & 18.533 & $\mathrm{C}_{10} \mathrm{H}_{18} \mathrm{O}$ & 154 \\
\hline 18. & Terpin-4-ol & 0.1650 & 1194 & 19.890 & $\mathrm{C}_{10} \mathrm{H}_{18} \mathrm{O}$ & 154 \\
\hline 19. & $\alpha$-Terpineol & 0.1844 & 1209 & 20.609 & $\mathrm{C}_{10} \mathrm{H}_{18} \mathrm{O}$ & 154 \\
\hline 20. & Decanal & 0.1722 & 1220 & 21.081 & $\mathrm{C}_{10} \mathrm{H}_{20} \mathrm{O}$ & 156 \\
\hline 21. & Thymol methyl ether & 0.1356 & 1244 & 22.168 & $\mathrm{C}_{11} \mathrm{H}_{16} \mathrm{O}$ & 164 \\
\hline 22. & Neral & 0.0207 & 1251 & 22.551 & $\mathrm{C}_{10} \mathrm{H}_{16} \mathrm{O}$ & 152 \\
\hline 23. & $\alpha$-Citral & 0.0308 & 1281 & 23.924 & $\mathrm{C}_{10} \mathrm{H}_{16} \mathrm{O}$ & 152 \\
\hline 24. & 2-Decyn-1-ol & 0.0323 & 1287 & 24.234 & $\mathrm{C}_{10} \mathrm{H}_{18} \mathrm{O}$ & 154 \\
\hline 25 . & Thymol & 0.0909 & 1307 & 25.251 & $\mathrm{C}_{10} \mathrm{H}_{14} \mathrm{O}$ & 150 \\
\hline 26. & $\alpha$-Elemene & 0.0269 & 1349 & 26.914 & $\mathrm{C}_{15} \mathrm{H}_{24}$ & 204 \\
\hline 27. & $\beta$-Elemene & 0.0151 & 1403 & 29.304 & $\mathrm{C}_{15} \mathrm{H}_{24}$ & 204 \\
\hline 28. & Dodecanal & 0.0198 & 1424 & 30.191 & $\mathrm{C}_{12} \mathrm{H}_{24} \mathrm{O}$ & 184 \\
\hline 29. & Bicyclogermacrene & 0.0180 & 1443 & 31.004 & $\mathrm{C}_{15} \mathrm{H}_{24}$ & 204 \\
\hline 30. & $\beta$-Farnesene & 0.0192 & 1467 & 32.005 & $\mathrm{C}_{15} \mathrm{H}_{24}$ & 204 \\
\hline 31. & Germacrene D & 0.0539 & 1493 & 33.143 & $\mathrm{C}_{15} \mathrm{H}_{24}$ & 204 \\
\hline 32. & $\alpha$-Farnesene & 0.0179 & 1519 & 34.139 & $\mathrm{C}_{15} \mathrm{H}_{24}$ & 204 \\
\hline 33. & Germacrene B & 0.0201 & 1571 & 36.271 & $\mathrm{C}_{15} \mathrm{H}_{24}$ & 204 \\
\hline
\end{tabular}

Table 4c. Composition of essential oil compounds at 1200-1400m altitude in mature stage.

\begin{tabular}{|c|c|c|c|c|c|c|}
\hline S. N. & Compound name & Area \% & $\overline{\mathbf{R I}}$ & R. Time & $\begin{array}{l}\text { Molecular } \\
\text { formula }\end{array}$ & $\begin{array}{l}\text { Molecular } \\
\text { weight }\end{array}$ \\
\hline 1. & $\alpha$-Thujene & 0.1335 & 922 & 8.449 & $\mathrm{C}_{10} \mathrm{H}_{16}$ & 136 \\
\hline 2. & $\alpha$-Pinene & 0.6222 & 929 & 8.735 & $\mathrm{C}_{10} \mathrm{H}_{16}$ & 136 \\
\hline 3. & Camphene & 0.0321 & 945 & 9.376 & $\mathrm{C}_{10} \mathrm{H}_{16}$ & 136 \\
\hline 4. & Sabinene & 0.5046 & 969 & 10.331 & $\mathrm{C}_{10} \mathrm{H}_{16}$ & 136 \\
\hline 5. & $\beta$-Pinene & 0.4074 & 973 & 10.527 & $\mathrm{C}_{10} \mathrm{H}_{16}$ & 136 \\
\hline 6. & Myrcene & 1.5654 & 988 & 11.071 & $\mathrm{C}_{10} \mathrm{H}_{16}$ & 136 \\
\hline 7. & Octanal & 0.3272 & 1003 & 11.668 & $\mathrm{C}_{8} \mathrm{H}_{16} \mathrm{O}$ & 128 \\
\hline 8. & $\alpha$-Terpinene & 0.0902 & 1015 & 12.281 & $\mathrm{C}_{10} \mathrm{H}_{16}$ & 136 \\
\hline 9. & Limonene & 87.2034 & 1042 & 13.263 & $\mathrm{C}_{10} \mathrm{H}_{16}$ & 136 \\
\hline 10. & $\beta$-Ocimene & 0.0837 & 1048 & 13.674 & $\mathrm{C}_{10} \mathrm{H}_{16}$ & 136 \\
\hline 11. & Y -Terpinene & 4.8204 & 1062 & 14.265 & $\mathrm{C}_{10} \mathrm{H}_{16}$ & 136 \\
\hline 12. & 1-Octanol & 0.0993 & 1072 & 14.832 & $\mathrm{C}_{8} \mathrm{H}_{18} \mathrm{O}$ & 130 \\
\hline 13. & a -Terpinolene & 0.2396 & 1085 & 15.423 & $\mathrm{C}_{10} \mathrm{H}_{16}$ & 136 \\
\hline 14. & $\alpha-p$-dimethylstyrene & 0.0324 & 1089 & 15.654 & $\mathrm{C}_{10} \mathrm{H}_{12}$ & 132 \\
\hline 15. & Linalool & 1.5009 & 1101 & 16.209 & $\mathrm{C}_{10} \mathrm{H}_{18} \mathrm{O}$ & 154 \\
\hline 16. & Nonanal & 0.0347 & 1104 & 16.340 & $\mathrm{C}_{9} \mathrm{H}_{18} \mathrm{O}$ & 142 \\
\hline 17. & trans-p-Mentha-2,8-dienol & 0.0080 & 1120 & 17.164 & $\mathrm{C}_{10} \mathrm{H}_{16} \mathrm{O}$ & 152 \\
\hline 18. & Cis-Mentha-2,8-dien-1-ol & 0.0237 & 1130 & 17.834 & $\mathrm{C}_{10} \mathrm{H}_{16} \mathrm{O}$ & 152 \\
\hline 19. & Citronellal & 0.1021 & 1135 & 18.546 & $\mathrm{C}_{10} \mathrm{H}_{18} \mathrm{O}$ & 154 \\
\hline 20. & Terpinen-4-ol & 0.1905 & 1138 & 19.904 & $\mathrm{C}_{10} \mathrm{H}_{18} \mathrm{O}$ & 154 \\
\hline 21. & $\alpha$-Terpineol & 0.2335 & 1146 & 20.627 & $\mathrm{C}_{10} \mathrm{H}_{18} \mathrm{O}$ & 154 \\
\hline 22. & Decanal & 0.1800 & 1151 & 21.096 & $\mathrm{C}_{10} \mathrm{H}_{20} \mathrm{O}$ & 156 \\
\hline 23. & Trans-Carveol & 0.0209 & 1178 & 21.762 & $\mathrm{C}_{10} \mathrm{H}_{16} \mathrm{O}$ & 152 \\
\hline 24. & Nerol & 0.0141 & 1186 & 21.990 & $\mathrm{C}_{10} \mathrm{H}_{16} \mathrm{O}$ & 152 \\
\hline 25. & Thymol methyl ether & 0.5752 & 1194 & 22.200 & $\mathrm{C}_{11} \mathrm{H}_{16} \mathrm{O}$ & 164 \\
\hline 26. & Neral & 0.0254 & 1205 & 22.570 & $\mathrm{C}_{10} \mathrm{H}_{16} \mathrm{O}$ & 152 \\
\hline 27. & Carvone & 0.0345 & 1219 & 22.823 & $\mathrm{C}_{10} \mathrm{H}_{14} \mathrm{O}$ & 150 \\
\hline 28. & Geraniol & 0.0183 & 1231 & 23.200 & $\mathrm{C}_{10} \mathrm{H}_{18} \mathrm{O}$ & 154 \\
\hline 29. & 2,6-Octadienal, 3,7-dimethyl-, (E)- & 0.0496 & 1238 & 23.946 & $\mathrm{C}_{10} \mathrm{H}_{16} \mathrm{O}$ & 152 \\
\hline 30. & Perillaldehyde & 0.0470 & 1242 & 24.250 & $\mathrm{C}_{10} \mathrm{H}_{14} \mathrm{O}$ & 150 \\
\hline 31. & Bornyl acetate & 0.0455 & 1251 & 24.638 & $\mathrm{C}_{12} \mathrm{H}_{20} \mathrm{O}_{2}$ & 196 \\
\hline 32. & Carvacrol & 0.1657 & 1266 & 25.270 & $\mathrm{C}_{10} \mathrm{H}_{14} \mathrm{O}$ & 150 \\
\hline 33. & Undecanal & 0.0190 & 1272 & 25.818 & $\mathrm{C}_{11} \mathrm{H}_{22} \mathrm{O}$ & 170 \\
\hline 34. & a-Elemene & 0.0373 & 1289 & 26.925 & $\mathrm{C}_{15} \mathrm{H}_{24}$ & 204 \\
\hline 35. & Geranyl acetate & 0.0077 & 1293 & 28.928 & $\mathrm{C}_{12} \mathrm{H}_{20} \mathrm{O}_{2}$ & 196 \\
\hline 36. & $\beta$-Elemene & 0.0197 & 1306 & 29.322 & $\mathrm{C}_{15} \mathrm{H}_{24}$ & 204 \\
\hline 37. & Dodecanal & 0.0232 & 1319 & 30.214 & $\mathrm{C}_{12} \mathrm{H}_{24} \mathrm{O}$ & 128 \\
\hline 38. & Caryophyllene <(E)-> & 0.0191 & 1332 & 30.580 & $\mathrm{C}_{15} \mathrm{H}_{24}$ & 204 \\
\hline 39. & $\mathrm{y}$-Elemene & 0.0110 & 1338 & 31.021 & $\mathrm{C}_{15} \mathrm{H}_{24}$ & 204 \\
\hline 40. & Cis- $\alpha$-Bergamotene & 0.0283 & 1344 & 31.179 & $\mathrm{C}_{15} \mathrm{H}_{24}$ & 204 \\
\hline 41. & $\beta$-Farnesene & 0.0361 & 1349 & 32.025 & $\mathrm{C}_{15} \mathrm{H}_{24}$ & 204 \\
\hline 42. & Germacrene D & 0.0679 & 1415 & 33.168 & $\mathrm{C}_{15} \mathrm{H}_{24}$ & 204 \\
\hline 43. & Bicyclogermacrene & 0.0119 & 1430 & 33.772 & $\mathrm{C}_{15} \mathrm{H}_{24}$ & 204 \\
\hline 44. & $\alpha-F a r n e s e n e$ & 0.0173 & 1476 & 34.161 & $\mathrm{C}_{15} \mathrm{H}_{24}$ & 204 \\
\hline 45. & $\beta$-Bisabolene & 0.0406 & 1502 & 34.296 & $\mathrm{C}_{15} \mathrm{H}_{24}$ & 204 \\
\hline 46. & Trans- $\beta$-Bergamotene & 0.0086 & 1505 & 34.932 & $\mathrm{C}_{15} \mathrm{H}_{24}$ & 204 \\
\hline 47. & Germacrene B & 0.0385 & 1553 & 36.294 & $\mathrm{C}_{15} \mathrm{H}_{24}$ & 204 \\
\hline 48. & a-Bisabolene & 0.0107 & 1624 & 38.788 & $\mathrm{C}_{15} \mathrm{H}_{24} \mathrm{O}$ & 220 \\
\hline 49. & a-Cadinol & 0.0723 & 1659 & 39.328 & $\mathrm{C}_{15} \mathrm{H}_{26} \mathrm{O}$ & 222 \\
\hline 50. & a-Bisabolol & 0.0334 & 1668 & 41.475 & $\mathrm{C}_{15} \mathrm{H}_{26} \mathrm{O}$ & 222 \\
\hline 51. & a-Sinensal & 0.0665 & 1684 & 43.519 & $\mathrm{C}_{15} \mathrm{H}_{22} \mathrm{O}$ & 218 \\
\hline
\end{tabular}


Pradhan, A. et al. / J. Appl. \& Nat. Sci. 11(1): 168-181 (2019)

Table 4d. Composition of essential oil compounds at 1400-1600m altitude in mature stage.

\begin{tabular}{|c|c|c|c|c|c|c|}
\hline S.N. & Compound name & Area \% & $\mathbf{R I}$ & R. Time & $\begin{array}{l}\text { Molecular } \\
\text { formula }\end{array}$ & $\begin{array}{l}\text { Molecular } \\
\text { weight }\end{array}$ \\
\hline 1. & $\alpha$-Thujene & 0.1375 & 923 & 8.443 & $\mathrm{C}_{10} \mathrm{H}_{16}$ & 136 \\
\hline 2. & $\alpha$-Pinene & 0.6000 & 930 & 8.727 & $\mathrm{C}_{10} \mathrm{H}_{16}$ & 136 \\
\hline 3. & Camphene & 0.1883 & 945 & 9.369 & $\mathrm{C}_{10} \mathrm{H}_{16}$ & 136 \\
\hline 4. & Sabinene & 0.6698 & 969 & 10.323 & $\mathrm{C}_{10} \mathrm{H}_{16}$ & 136 \\
\hline 5. & $\beta$-Pinene & 0.7190 & 974 & 10.516 & $\mathrm{C}_{10} \mathrm{H}_{16}$ & 136 \\
\hline 6. & Myrcene & 1.4426 & 988 & 11.053 & $\mathrm{C}_{10} \mathrm{H}_{16}$ & 136 \\
\hline 7. & $\alpha$-Terpinene & 0.0737 & 997 & 11.450 & $\mathrm{C}_{10} \mathrm{H}_{16}$ & 136 \\
\hline 8. & Octanal & 0.6826 & 1003 & 11.665 & $\mathrm{C}_{8} \mathrm{H}_{16} \mathrm{O}$ & 128 \\
\hline 9. & $\alpha$-Humulene & 0.1104 & 1015 & 12.258 & $\mathrm{C}_{10} \mathrm{H}_{16}$ & 136 \\
\hline 10. & Limonene & 73.0895 & 1035 & 13.122 & $\mathrm{C}_{10} \mathrm{H}_{16}$ & 136 \\
\hline 11. & Phenylacetaldehyde & 0.0218 & 1041 & 13.505 & $\mathrm{C}_{8} \mathrm{H}_{8} \mathrm{O}$ & 120 \\
\hline 12. & $\beta$-Ocimene & 0.1086 & 1046 & 13.633 & $\mathrm{C}_{10} \mathrm{H}_{16}$ & 136 \\
\hline 13. & Y -Terpinene & 4.4602 & 1058 & 14.205 & $\mathrm{C}_{10} \mathrm{H}_{16}$ & 136 \\
\hline 14. & Cis-Linalool oxide & 0.0590 & 1069 & 14.721 & $\mathrm{C}_{10} \mathrm{H}_{18} \mathrm{O}_{2}$ & 170 \\
\hline 15. & 1-Octanol & 0.2106 & 1073 & 14.848 & $\mathrm{C}_{8} \mathrm{H}_{18} \mathrm{O}$ & 130 \\
\hline 16. & $\alpha$-Terpinolene & 0.2570 & 1083 & 15.400 & $\mathrm{C}_{10} \mathrm{H}_{16}$ & 136 \\
\hline 17. & Linalool & 9.1389 & 1105 & 16.305 & $\mathrm{C}_{10} \mathrm{H}_{18} \mathrm{O}$ & 154 \\
\hline 18. & Trans-p-Mentha-2,8-dien-1-ol & 0.0379 & 1121 & 17.279 & $\mathrm{C}_{10} \mathrm{H}_{16} \mathrm{O}$ & 152 \\
\hline 19. & (Z) Sabinene Hydrate & 0.0242 & 1124 & 17.608 & $\mathrm{C}_{10} \mathrm{H}_{18} \mathrm{O}$ & 154 \\
\hline 20. & Cis-p-Mentha-2,8-dien-1-ol & 0.0398 & 1135 & 17.843 & $\mathrm{C}_{10} \mathrm{H}_{16} \mathrm{O}$ & 152 \\
\hline 21. & $\beta$-Terpineol & 0.0371 & 1141 & 18.125 & $\mathrm{C}_{10} \mathrm{H}_{18} \mathrm{O}$ & 154 \\
\hline 22. & Citronellal & 0.0562 & 1151 & 18.536 & $\mathrm{C}_{10} \mathrm{H}_{18} \mathrm{O}$ & 154 \\
\hline 23. & Borneol & 0.1012 & 1170 & 19.529 & $\mathrm{C}_{10} \mathrm{H}_{18} \mathrm{O}$ & 154 \\
\hline 24. & Terpinen-4-ol & 1.8127 & 1180 & 19.942 & $\mathrm{C}_{10} \mathrm{H}_{18} \mathrm{O}$ & 154 \\
\hline 25. & p-Cymen-9-ol & 0.0972 & 1187 & 20.294 & $\mathrm{C}_{10} \mathrm{H}_{18} \mathrm{O}$ & 154 \\
\hline 26. & a -Terpineol & 1.3312 & 1196 & 20.662 & $\mathrm{C}_{10} \mathrm{H}_{18} \mathrm{O}$ & 154 \\
\hline 27. & Decanal & 0.2439 & 1206 & 21.093 & $\mathrm{C}_{10} \mathrm{H}_{20} \mathrm{O}$ & 156 \\
\hline 28. & Trans-Carveol & 0.0317 & 1218 & 21.763 & $\mathrm{C}_{10} \mathrm{H}_{16} \mathrm{O}$ & 152 \\
\hline 29. & 2,6-Octadien-1-ol & 0.1005 & 1224 & 21.986 & $\mathrm{C}_{10} \mathrm{H}_{18} \mathrm{O}$ & 154 \\
\hline 30. & Carvacryl methyl ether & 0.2813 & 1228 & 22.170 & $\mathrm{C}_{11} \mathrm{H}_{16} \mathrm{O}$ & 164 \\
\hline 31. & Neral & 0.0747 & 1237 & 22.566 & $\mathrm{C}_{10} \mathrm{H}_{16} \mathrm{O}$ & 152 \\
\hline 32. & Carvone & 0.0458 & 1241 & 22.814 & $\mathrm{C}_{10} \mathrm{H}_{14} \mathrm{O}$ & 150 \\
\hline 33. & Geraniol & 0.1200 & 1251 & 23.205 & $\mathrm{C}_{10} \mathrm{H}_{18} \mathrm{O}$ & 154 \\
\hline 34. & Geranial & 0.1439 & 1267 & 23.945 & $\mathrm{C}_{10} \mathrm{H}_{16} \mathrm{O}$ & 152 \\
\hline 35. & Perillaldehyde & 0.1053 & 1272 & 24.247 & $\mathrm{C}_{10} \mathrm{H}_{14} \mathrm{O}$ & 150 \\
\hline 36. & Bornyl acetate & 0.3379 & 1282 & 24.637 & $\mathrm{C}_{12} \mathrm{H}_{20} \mathrm{O}_{2}$ & 196 \\
\hline 37. & Limonen-10-ol & 0.0472 & 1290 & 25.063 & $\mathrm{C}_{10} \mathrm{H}_{16} \mathrm{O}$ & 152 \\
\hline 38. & Thymol & 1.2160 & 1295 & 25.295 & $\mathrm{C}_{10} \mathrm{H}_{14} \mathrm{O}$ & 150 \\
\hline 39. & 1,3-Dioxolane, 2,2-dimethyl-4,5-dipropenyl & 0.0342 & 1304 & 25.686 & $\mathrm{C}_{11} \mathrm{H}_{18} \mathrm{O}_{2}$ & 182 \\
\hline 40. & Cyclohexene & 0.1087 & 1322 & 26.430 & $\mathrm{C}_{11} \mathrm{H}_{20} \mathrm{O}$ & 168 \\
\hline 41. & Neoisopulegol hydrate & 0.0431 & 1339 & 27.264 & $\mathrm{C}_{10} \mathrm{H}_{20} \mathrm{O}_{2}$ & 172 \\
\hline 42. & Limonene glycol & 0.1286 & 1346 & 27.566 & $\mathrm{C}_{10} \mathrm{H}_{18} \mathrm{O}_{2}$ & 170 \\
\hline 43. & Carvotanacetone<8-hydroxy-> & 0.0410 & 1400 & 29.974 & $\mathrm{C}_{10} \mathrm{H}_{16} \mathrm{O}_{2}$ & 168 \\
\hline 44. & 2-Cyclohexen-1-one & 0.0404 & 1423 & 30.984 & $\mathrm{C}_{10} \mathrm{H}_{16} \mathrm{O}_{2}$ & 168 \\
\hline 45. & $\beta$-Farnesene & 0.0650 & 1452 & 32.012 & $\mathrm{C}_{15} \mathrm{H}_{24}$ & 204 \\
\hline 46. & Germacrene D & 0.1128 & 1476 & 33.161 & $\mathrm{C}_{15} \mathrm{H}_{24}$ & 204 \\
\hline 47. & 2 Methyl isoborneol & 0.0874 & 1492 & 33.808 & $\mathrm{C}_{11} \mathrm{H}_{20} \mathrm{O}$ & 168 \\
\hline 48. & $\beta$-Bisabolene & 0.0812 & 1505 & 34.288 & $\mathrm{C}_{15} \mathrm{H}_{24}$ & 204 \\
\hline 49. & $\alpha$-Elemol & 0.0282 & 1545 & 35.940 & $\mathrm{C}_{15} \mathrm{H}_{26} \mathrm{O}$ & 222 \\
\hline 50 & Spathulenol & 0.0246 & 1572 & 37.057 & $\mathrm{C}_{15} \mathrm{H}_{24} \mathrm{O}$ & 220 \\
\hline 51. & trans-Valerenyl acetate & 0.0227 & 1618 & 38.786 & $\mathrm{C}_{17} \mathrm{H}_{26} \mathrm{O}_{2}$ & 262 \\
\hline 52. & a-Muurolol & 0.4692 & 1633 & 39.332 & $\mathrm{C}_{15} \mathrm{H}_{26} \mathrm{O}$ & 222 \\
\hline 53. & Cadin-4-en-10-ol & 0.0720 & 1651 & 40.066 & $\mathrm{C}_{15} \mathrm{H}_{26} \mathrm{O}$ & 222 \\
\hline 54. & $\alpha$-Bisabolol & 0.1471 & 1685 & 41.304 & $\mathrm{C}_{15} \mathrm{H}_{26} \mathrm{O}$ & 222 \\
\hline 55. & Zierone & 0.1438 & 1689 & 41.468 & $\mathrm{C}_{15} \mathrm{H}_{22} \mathrm{O}$ & 218 \\
\hline 56. & a-Sinensal & 0.0948 & 1746 & 43.511 & $\mathrm{C}_{15} \mathrm{H}_{22} \mathrm{O}$ & 218 \\
\hline
\end{tabular}


Pradhan, A. et al. / J. Appl. \& Nat. Sci. 11(1): 168-181 (2019)

Table 4e. Composition of essential oil compounds at $>1600 \mathrm{~m}$ altitude in mature stage.

\begin{tabular}{|c|c|c|c|c|c|c|}
\hline S. $\mathbf{N}$. & Compound name & Area \% & $\mathbf{R I}$ & R. Time & $\begin{array}{l}\text { Molecular } \\
\text { formula }\end{array}$ & $\begin{array}{l}\text { Molecular } \\
\text { weight }\end{array}$ \\
\hline 1. & $\alpha$-Thujene & 0.1417 & 923 & 8.458 & $\mathrm{C}_{10} \mathrm{H}_{16}$ & 136 \\
\hline 2. & $\alpha$-Pinene & 0.6977 & 930 & 8.751 & $\mathrm{C}_{10} \mathrm{H}_{16}$ & 136 \\
\hline 3. & Camphene & 0.0053 & 945 & 9.384 & $\mathrm{C}_{10} \mathrm{H}_{16}$ & 136 \\
\hline 4. & Sabinene & 0.7037 & 969 & 10.351 & $\mathrm{C}_{10} \mathrm{H}_{16}$ & 136 \\
\hline 5. & $\beta$-Pinene & 0.4439 & 974 & 10.545 & $\mathrm{C}_{10} \mathrm{H}_{16}$ & 136 \\
\hline 6. & Myrcene & 1.6085 & 989 & 11.096 & $\mathrm{C}_{10} \mathrm{H}_{16}$ & 136 \\
\hline 7. & Octanal & 0.3542 & 1003 & 11.698 & $\mathrm{C}_{8} \mathrm{H}_{16} \mathrm{O}$ & 128 \\
\hline 8. & $(+)$-2-Carene & 0.0214 & 1015 & 12.280 & $\mathrm{C}_{10} \mathrm{H}_{16}$ & 136 \\
\hline 9. & y -Terpinyl acetate & 0.0559 & 1022 & 12.661 & $\mathrm{C}_{12} \mathrm{H}_{20} \mathrm{O}_{2}$ & 196 \\
\hline 10. & Limonene & 88.4631 & 1042 & 13.604 & $\mathrm{C}_{10} \mathrm{H}_{16}$ & 136 \\
\hline 11. & $\beta$-Ocimene & 0.0691 & 1048 & 13.818 & $\mathrm{C}_{10} \mathrm{H}_{16}$ & 136 \\
\hline 12. & Y -Terpinene & 3.9952 & 1061 & 14.422 & $\mathrm{C}_{10} \mathrm{H}_{16}$ & 136 \\
\hline 13. & 1-Octanol & 0.1646 & 1072 & 14.908 & $\mathrm{C}_{8} \mathrm{H}_{18} \mathrm{O}$ & 130 \\
\hline 14. & $\alpha$-Terpinolene & 0.1899 & 1085 & 15.482 & $\mathrm{C}_{10} \mathrm{H}_{16}$ & 136 \\
\hline 15. & $\alpha-p$-Dimethylstyrene & 0.0138 & 1089 & 15.698 & $\mathrm{C}_{10} \mathrm{H}_{12}$ & 132 \\
\hline 16. & Linalool & 1.6725 & 1103 & 16.321 & $\mathrm{C}_{10} \mathrm{H}_{18} \mathrm{O}$ & 154 \\
\hline 17. & trans-p-Mentha-2,8-dienol & 0.0067 & 1121 & 17.196 & $\mathrm{C}_{10} \mathrm{H}_{16} \mathrm{O}$ & 152 \\
\hline 18. & Cis-Limonene oxide & 0.0148 & 1131 & 17.637 & $\mathrm{C}_{10} \mathrm{H}_{16} \mathrm{O}$ & 152 \\
\hline 19. & Trans-Limonene oxide & 0.0247 & 1135 & 17.852 & $\mathrm{C}_{10} \mathrm{H}_{16} \mathrm{O}$ & 152 \\
\hline 20. & Citronellal & 0.0819 & 1151 & 18.561 & $\mathrm{C}_{10} \mathrm{H}_{18} \mathrm{O}$ & 154 \\
\hline 21. & 1-Nonanol & 0.0113 & 1172 & 19.567 & $\mathrm{C}_{9} \mathrm{H}_{20} \mathrm{O}$ & 144 \\
\hline 22. & Terpinen-4-ol & 0.1563 & 1179 & 19.921 & $\mathrm{C}_{10} \mathrm{H}_{18} \mathrm{O}$ & 154 \\
\hline 23. & m-Cymen-8-ol & 0.0067 & 1186 & 20.292 & $\mathrm{C}_{10} \mathrm{H}_{14} \mathrm{O}$ & 152 \\
\hline 24. & $\alpha$-Terpineol & 0.2111 & 1194 & 20.649 & $\mathrm{C}_{10} \mathrm{H}_{18} \mathrm{O}$ & 150 \\
\hline 25. & Decanal & 0.2305 & 1206 & 21.116 & $\mathrm{C}_{10} \mathrm{H}_{20} \mathrm{O}$ & 156 \\
\hline 26. & Nerol & 0.0106 & 1224 & 21.992 & $\mathrm{C}_{10} \mathrm{H}_{18} \mathrm{O}$ & 154 \\
\hline 27. & Thymol methyl ether & 0.1379 & 1228 & 22.189 & $\mathrm{C}_{11} \mathrm{H}_{16} \mathrm{O}$ & 164 \\
\hline 28. & Neral & 0.0144 & 1236 & 22.566 & $\mathrm{C}_{10} \mathrm{H}_{16} \mathrm{O}$ & 152 \\
\hline 29. & Carvone & 0.0160 & 1241 & 22.818 & $\mathrm{C}_{10} \mathrm{H}_{14} \mathrm{O}$ & 150 \\
\hline 30. & Geranial & 0.0270 & 1266 & 23.939 & $\mathrm{C}_{10} \mathrm{H}_{16} \mathrm{O}$ & 152 \\
\hline 31. & Perillaldehyde & 0.0527 & 1272 & 24.247 & $\mathrm{C}_{10} \mathrm{H}_{14} \mathrm{O}$ & 150 \\
\hline 32. & Thymol & 0.1871 & 1293 & 25.273 & $\mathrm{C}_{10} \mathrm{H}_{14} \mathrm{O}$ & 150 \\
\hline 33. & Undecanal & 0.0049 & 1307 & 25.752 & $\mathrm{C}_{11} \mathrm{H}_{22} \mathrm{O}$ & 170 \\
\hline 34. & $\alpha$-Elemene & 0.0131 & 1333 & 26.921 & $\mathrm{C}_{15} \mathrm{H}_{24}$ & 204 \\
\hline 35. & $\beta$-Elemene & 0.0138 & 1387 & 29.312 & $\mathrm{C}_{15} \mathrm{H}_{24}$ & 204 \\
\hline 36. & Dodecanal & 0.0238 & 1408 & 30.199 & $\mathrm{C}_{12} \mathrm{H}_{24} \mathrm{O}$ & 180 \\
\hline 37. & $\mathrm{y}$-Elemene & 0.0156 & 1426 & 31.011 & $\mathrm{C}_{15} \mathrm{H}_{24}$ & 204 \\
\hline 38. & $\beta$-Farnesene & 0.0200 & 1451 & 32.012 & $\mathrm{C}_{15} \mathrm{H}_{24}$ & 204 \\
\hline 39. & Germacrene D & 0.0375 & 1476 & 33.155 & $\mathrm{C}_{15} \mathrm{H}_{24}$ & 204 \\
\hline 40. & a-Farnesene & 0.0189 & 1502 & 34.149 & $\mathrm{C}_{15} \mathrm{H}_{24}$ & 204 \\
\hline 41. & Germacrene B & 0.0134 & 1553 & 36.284 & $\mathrm{C}_{15} \mathrm{H}_{24}$ & 204 \\
\hline 42. & $\beta$-Sinensal & 0.0169 & 1690 & 41.474 & $\mathrm{C}_{15} \mathrm{H}_{24}$ & 204 \\
\hline 43. & Trans- $\alpha$-Bergamotene & 0.0322 & 1747 & 43.512 & $\mathrm{C}_{15} \mathrm{H}_{24}$ & 204 \\
\hline
\end{tabular}

are mentioned in Table2. It showed sharp and strong peaks at 3607 at $800 \mathrm{~m}$ altitude with $\mathrm{O}-\mathrm{H}$ stretch. C-H stretch was found to be $2956 \mathrm{~cm}^{-1}$ at $800-1000 \mathrm{~m}$ altitude. Further $\mathrm{C}=\mathrm{O}$ bond was found at $1727 \mathrm{~cm}^{-1}$ at $800-1000 \mathrm{~m}$ altitude was observed the highest value which showed $\alpha, \beta$ unsaturated ester. C-N stretch with aliphatic amines was recorded in 1075, 1201 at 800m. Likewise, 1073, $1219(1000-1200 \mathrm{~m})$. Further $\left(1074 \mathrm{~cm}^{-1}, 1214 \mathrm{~cm}^{-}\right.$ $\left.{ }_{1}^{1}\right) ;\left(1017 \mathrm{~cm}^{-1}, 1064 \mathrm{~cm}^{-1}\right)$ and $\left(1021 \mathrm{~cm}^{-1}, 1095 \mathrm{~cm}^{-}\right.$ $\left.{ }^{1}\right)$ were observed in $1200-1400 \mathrm{~m}, 1400-1600 \mathrm{~m}$ and $>1600 \mathrm{~m}$ altitudes, respectively. In addition alkyl halides was noted only in 1400-1600m (1103 $\mathrm{cm}^{-1}$ ) and $>1600 \mathrm{~m}\left(1108 \mathrm{~cm}^{-1}\right)$ as $\mathrm{C}-\mathrm{H}$ wag. It is compared with Silva et al., 2012 who mentioned the frequency as carbohydrate ring. The result in the present finding can be compared with Kanmani et al., 2014 who performed research in C. limon. In their research sharp and strong peaks at $3595.31 \mathrm{~cm}^{-1}$ as $\mathrm{O}-\mathrm{H}$ stretch, $\mathrm{C}-\mathrm{H}$ stretch in the frequency 2830-2695 $\mathrm{cm}^{-1}$ and strong $\mathrm{C}=\mathrm{O}$ unsaturated esters and aliphatic amine functional stretch occurring at $1710-1665 \mathrm{~cm}^{-1}$

Instrumental analysis: GCMS analysis chromatograms and table are presented in Table $5 a, 5 b, 5 c, 5 d, 5$.Inmature stage, five different altitudes showed compound identification as $39,33,51,56,43$ at $800 \mathrm{~m}, 1000-1200 \mathrm{~m}, 1200-$ $1400 \mathrm{~m}, 1400-1600 \mathrm{~m}$ and $>1600 \mathrm{~m}$ altitudes. Limonene content was found to be the highest in the altitudes viz:800m, 1000-1200m, 1200-1400m, $1400-1600 \mathrm{~m}$ and $>1600 \mathrm{~m}(88.40,87.64,87.20$, 
Pradhan, A. et al. / J. Appl. \& Nat. Sci. 11(1): 168-181 (2019)

Table 5a. Composition of essential oil compounds at $800 \mathrm{~m}$ altitude in immature stage.

\begin{tabular}{|c|c|c|c|c|c|c|}
\hline S.N. & Compound name & Area \% & $\mathbf{R I}$ & R. Time & $\begin{array}{l}\text { Molecular } \\
\text { formula }\end{array}$ & $\begin{array}{l}\text { Molecular } \\
\text { weight }\end{array}$ \\
\hline 1. & $\alpha$-Thujene & 0.1189 & 922 & 8.433 & $\mathrm{C}_{10} \mathrm{H}_{16}$ & 136 \\
\hline 2. & $\alpha$-Pinene & 0.6590 & 929 & 8.718 & $\mathrm{C}_{10} \mathrm{H}_{16}$ & 136 \\
\hline 3. & Sabinene & 0.1778 & 968 & 10.306 & $\mathrm{C}_{10} \mathrm{H}_{16}$ & 136 \\
\hline 4. & $\beta$-Pinene & 0.3613 & 973 & 10.503 & $\mathrm{C}_{10} \mathrm{H}_{16}$ & 136 \\
\hline 5. & Myrcene & 1.6191 & 988 & 11.042 & $\mathrm{C}_{10} \mathrm{H}_{16}$ & 136 \\
\hline 6. & Octanal & 0.1210 & 1002 & 11.632 & $\mathrm{C}_{8} \mathrm{H}_{16} \mathrm{O}$ & 128 \\
\hline 7. & Nonanal & 0.0373 & 1004 & 11.749 & $\mathrm{C}_{8} \mathrm{H}_{16} \mathrm{O}$ & 128 \\
\hline 8. & a -Terpinene & 0.1092 & 1014 & 12.248 & $\mathrm{C}_{10} \mathrm{H}_{16}$ & 136 \\
\hline 9. & y -Terpineol acetate & 0.1288 & 1023 & 12.661 & $\mathrm{C}_{10} \mathrm{H}_{16}$ & 136 \\
\hline 10. & Limonene & 87.9324 & 1045 & 13.163 & $\mathrm{C}_{10} \mathrm{H}_{16}$ & 136 \\
\hline 11. & $\beta$-Ocimene & 0.0367 & 1050 & 13.628 & $\mathrm{C}_{10} \mathrm{H}_{16}$ & 136 \\
\hline 12. & Y -Terpinene & 5.3748 & 1063 & 14.207 & $\mathrm{C}_{10} \mathrm{H}_{16}$ & 136 \\
\hline 13. & 1-Octanol & 0.0580 & 1071 & 14.786 & $\mathrm{C}_{10} \mathrm{H}_{16}$ & 136 \\
\hline 14. & $\alpha$-Terpinolene & 0.2604 & 1085 & 15.385 & $\mathrm{C}_{10} \mathrm{H}_{16}$ & 136 \\
\hline 15. & Linalool & 1.5219 & 1103 & 16.144 & $\mathrm{C}_{9} \mathrm{H}_{18} \mathrm{O}$ & 142 \\
\hline 16. & Nonanal & 0.0167 & 1105 & 16.298 & $\mathrm{C}_{9} \mathrm{H}_{18} \mathrm{O}$ & 142 \\
\hline 17. & Citronellal & 0.0120 & 1150 & 18.511 & $\mathrm{C}_{10} \mathrm{H}_{18} \mathrm{O}$ & 154 \\
\hline 18. & Terpinen-4-ol & 0.2172 & 1177 & 19.851 & $\mathrm{C}_{10} \mathrm{H}_{18} \mathrm{O}$ & 154 \\
\hline 19. & $\alpha$-Terpineol & 0.2697 & 1192 & 20.565 & $\mathrm{C}_{10} \mathrm{H}_{18} \mathrm{O}$ & 154 \\
\hline 20. & Decanal & 0.1916 & 1204 & 21.057 & $\mathrm{C}_{10} \mathrm{H}_{20} \mathrm{O}$ & 156 \\
\hline 21. & Trans-Carveol & 0.0120 & 1216 & 21.696 & $\mathrm{C}_{10} \mathrm{H}_{16} \mathrm{O}$ & 152 \\
\hline 22. & 2,6-Octadien-1-ol & 0.0215 & 1222 & 21.941 & $\mathrm{C}_{10} \mathrm{H}_{18} \mathrm{O}$ & 154 \\
\hline 23. & Thymol methyl ether & 0.0870 & 1227 & 22.137 & $\mathrm{C}_{11} \mathrm{H}_{16} \mathrm{O}$ & 164 \\
\hline 24. & Neral & 0.0143 & 1235 & 22.530 & $\mathrm{C}_{10} \mathrm{H}_{16} \mathrm{O}$ & 152 \\
\hline 25. & Geraniol & 0.0140 & 1248 & 23.157 & $\mathrm{C}_{10} \mathrm{H}_{18} \mathrm{O}$ & 154 \\
\hline 26. & Geranial & 0.0271 & 1264 & 23.910 & $\mathrm{C}_{10} \mathrm{H}_{16} \mathrm{O}$ & 152 \\
\hline 27. & Perillaldehyde & 0.0474 & 1270 & 24.204 & $\mathrm{C}_{10} \mathrm{H}_{14} \mathrm{O}$ & 150 \\
\hline 28. & Carvacrol & 0.2050 & 1289 & 25.159 & $\mathrm{C}_{10} \mathrm{H}_{14} \mathrm{O}$ & 150 \\
\hline 29. & Undecanal & 0.0072 & 1305 & 25.724 & $\mathrm{C}_{11} \mathrm{H}_{22} \mathrm{O}$ & 170 \\
\hline 30. & $\alpha$-Elemene & 0.0331 & 1331 & 26.891 & $\mathrm{C}_{15} \mathrm{H}_{24}$ & 204 \\
\hline 31. & $\beta$-Elemene & 0.0176 & 1385 & 29.276 & $\mathrm{C}_{15} \mathrm{H}_{24}$ & 204 \\
\hline 32. & Dodecanal & 0.0320 & 1406 & 30.169 & $\mathrm{C}_{12} \mathrm{H}_{24} \mathrm{O}$ & 184 \\
\hline 33. & $\mathrm{y}$-Elemene & 0.0060 & 1425 & 30.975 & $\mathrm{C}_{15} \mathrm{H}_{24}$ & 204 \\
\hline 34. & $\beta$-Farnesene & 0.0259 & 1450 & 31.985 & $\mathrm{C}_{15} \mathrm{H}_{24}$ & 204 \\
\hline 35. & Germacrene D & 0.0613 & 1474 & 33.111 & $\mathrm{C}_{15} \mathrm{H}_{24}$ & 204 \\
\hline 36. & a-Farnesene & 0.0224 & 1500 & 34.113 & $\mathrm{C}_{15} \mathrm{H}_{24}$ & 204 \\
\hline 37. & a-Cadinene & 0.0115 & 1512 & 34.660 & $\mathrm{C}_{15} \mathrm{H}_{24}$ & 204 \\
\hline 38. & Germacrene B & 0.0418 & 1551 & 36.239 & $\mathrm{C}_{15} \mathrm{H}_{24}$ & 204 \\
\hline 39. & $\beta$-Sinensal & 0.0353 & 1687 & 41.422 & $\mathrm{C}_{15} \mathrm{H}_{22} \mathrm{O}$ & 218 \\
\hline 40. & $\alpha$-Sinensal & 0.0537 & 1743 & 43.455 & $\mathrm{C}_{15} \mathrm{H}_{22} \mathrm{O}$ & 218 \\
\hline
\end{tabular}

Table 5b. Composition of essential oil compounds at 1000-1200m altitude in immature stage.

\begin{tabular}{|c|c|c|c|c|c|c|}
\hline $\begin{array}{l}\text { S. } \\
\text { No. }\end{array}$ & Compound name & Area \% & $\mathbf{R I}$ & $\begin{array}{l}\text { R. } \\
\text { Time }\end{array}$ & $\begin{array}{l}\text { Molecular } \\
\text { formula }\end{array}$ & $\begin{array}{l}\text { Molecular } \\
\text { weight }\end{array}$ \\
\hline 1. & $\alpha$-Pinene & 0.3816 & 928 & 8.709 & $\mathrm{C}_{10} \mathrm{H}_{16}$ & 136 \\
\hline 2. & Sabinene & 0.6908 & 967 & 10.298 & $\mathrm{C}_{10} \mathrm{H}_{16}$ & 136 \\
\hline 3. & $\beta$-Pinene & 0.4758 & 972 & 10.492 & $\mathrm{C}_{10} \mathrm{H}_{16}$ & 136 \\
\hline 4. & Myrcene & 1.5575 & 986 & 11.025 & $\mathrm{C}_{10} \mathrm{H}_{16}$ & 136 \\
\hline 5. & Octanal & 0.2045 & 1001 & 11.632 & $\mathrm{C}_{8} \mathrm{H}_{16} \mathrm{O}$ & 128 \\
\hline 6. & $\alpha$-Terpinene & 0.0395 & 1013 & 12.226 & $\mathrm{C}_{10} \mathrm{H}_{16}$ & 136 \\
\hline 7. & p-Cymene & 0.1511 & 1021 & 12.641 & $\mathrm{C}_{10} \mathrm{H}_{14}$ & 134 \\
\hline 8. & Limonene & 88.5797 & 1030 & 13.065 & $\mathrm{C}_{10} \mathrm{H}_{16}$ & 136 \\
\hline 9. & $\beta$-Ocimene & 0.0482 & 1043 & 13.595 & $\mathrm{C}_{10} \mathrm{H}_{16}$ & 136 \\
\hline 10. & Y -Terpinene & 1.4282 & 1054 & 14.124 & $\mathrm{C}_{10} \mathrm{H}_{16}$ & 136 \\
\hline 11. & Ethanone & 0.0264 & 1059 & 14.440 & $\mathrm{C}_{8} \mathrm{H}_{8} \mathrm{O}$ & 120 \\
\hline 12. & Cis- Linalool oxide & 0.3537 & 1067 & 14.684 & $\mathrm{C}_{10} \mathrm{H}_{18} \mathrm{O}_{2}$ & 170 \\
\hline 13. & Trans-Linaool oxide & 0.2340 & 1083 & 15.417 & $\mathrm{C}_{10} \mathrm{H}_{16} \mathrm{O}$ & 152 \\
\hline 14. & Linalool & 0.9189 & 1098 & 16.098 & $\mathrm{C}_{10} \mathrm{H}_{18} \mathrm{O}_{2}$ & 170 \\
\hline \multirow[t]{2}{*}{15.} & trans-p-Mentha-2,8-dienol & 0.1082 & 1118 & 17.095 & $\mathrm{C}_{10} \mathrm{H}_{16} \mathrm{O}$ & 152 \\
\hline & & & & & \multicolumn{2}{|c|}{ Contd........ } \\
\hline
\end{tabular}


Pradhan, A. et al. / J. Appl. \& Nat. Sci. 11(1): 168-181 (2019)

\begin{tabular}{|c|c|c|c|c|c|c|}
\hline 16. & Cyclopentan-1,2-dione & 0.0677 & 1123 & 17.493 & $\mathrm{C}_{7} \mathrm{H}_{10} \mathrm{O}_{2}$ & 126 \\
\hline 17. & Cis-p-Mentha-2,8-dien-1-ol & 0.1178 & 1132 & 17.782 & $\mathrm{C}_{10} \mathrm{H}_{16} \mathrm{O}$ & 152 \\
\hline 18. & Citronellal & 0.0304 & 1149 & 18.492 & $\mathrm{C}_{10} \mathrm{H}_{18} \mathrm{O}$ & 154 \\
\hline 19. & Terpinen-4-ol & 0.3719 & 1176 & 19.837 & $\mathrm{C}_{10} \mathrm{H}_{18} \mathrm{O}$ & 154 \\
\hline 20. & $\alpha$-Terpineol & 0.3013 & 1190 & 20.549 & $\mathrm{C}_{10} \mathrm{H}_{18} \mathrm{O}$ & 154 \\
\hline 21. & (-)-trans-Isopiperitenol & 0.0884 & 1195 & 20.776 & $\mathrm{C}_{10} \mathrm{H}_{16} \mathrm{O}$ & 152 \\
\hline 22. & Decanal & 0.0967 & 1203 & 21.040 & $\mathrm{C}_{10} \mathrm{H}_{20} \mathrm{O}$ & 156 \\
\hline 23. & Trans-carveol & 0.2577 & 1215 & 21.691 & $\mathrm{C}_{10} \mathrm{H}_{16} \mathrm{O}$ & 152 \\
\hline 24. & 2,6 Octadien-1-ol & 0.1550 & 1221 & 21.934 & $\mathrm{C}_{10} \mathrm{H}_{18} \mathrm{O}$ & 154 \\
\hline 25. & Cis-Carveol & 0.1412 & 1228 & 22.300 & $\mathrm{C}_{10} \mathrm{H}_{16} \mathrm{O}$ & 152 \\
\hline 26. & Carvone & 0.2026 & 1238 & 22.760 & $\mathrm{C}_{10} \mathrm{H}_{14} \mathrm{O}$ & 150 \\
\hline 27. & Geraniol & 0.1882 & 1248 & 23.142 & $\mathrm{C}_{10} \mathrm{H}_{18} \mathrm{O}$ & 154 \\
\hline 28. & $\alpha$-Citral & 0.0798 & 1263 & 23.891 & $\mathrm{C}_{10} \mathrm{H}_{16} \mathrm{O}$ & 152 \\
\hline 29. & Perillaldehyde & 0.0624 & 1269 & 24.196 & $\mathrm{C}_{10} \mathrm{H}_{14} \mathrm{O}$ & 150 \\
\hline 30. & 2-Cyclohexen-1-one & 0.0573 & 1288 & 25.153 & $\mathrm{C}_{10} \mathrm{H}_{14} \mathrm{O}$ & 150 \\
\hline 31. & p-Mentha-2,8-diene, 1-hydroperoxide & 0.2524 & 1302 & 25.743 & $\mathrm{C}_{10} \mathrm{H}_{16} \mathrm{O}_{2}$ & 168 \\
\hline 32. & $\alpha-S i n e n s a l$ & 0.2997 & 1315 & 26.333 & $\mathrm{C}_{15} \mathrm{H}_{22} \mathrm{O}$ & 218 \\
\hline 33. & Nerolidol & 0.1489 & 1326 & 26.804 & $\mathrm{C}_{15} \mathrm{H}_{26} \mathrm{O}$ & 222 \\
\hline 34. & Limonene-1,2-diol & 0.4984 & 1341 & 27.487 & $\mathrm{C}_{10} \mathrm{H}_{18} \mathrm{O}_{2}$ & 170 \\
\hline 35. & Patchenol & 0.1011 & 1348 & 27.774 & $\mathrm{C}_{11} \mathrm{H}_{18} \mathrm{O}$ & 166 \\
\hline 36. & $\alpha$-Farnsesene & 0.2574 & 1355 & 28.109 & $\mathrm{C}_{15} \mathrm{H}_{24}$ & 204 \\
\hline 37. & 2,6,6-Trimethyl-1-cyclohexene-1-carboxylic acid & 0.0346 & 1363 & 28.450 & $\mathrm{C}_{10} \mathrm{H}_{16} \mathrm{O}_{2}$ & 168 \\
\hline 38. & a-Sinensal & 0.2250 & 1372 & 28.836 & $\mathrm{C}_{15} \mathrm{H}_{22} \mathrm{O}$ & 218 \\
\hline 39. & Germacrene D & 0.4537 & 1474 & 33.101 & $\mathrm{C}_{15} \mathrm{H}_{24}$ & 204 \\
\hline 40. & Trans- $\alpha$-.Bergamotene & 0.0514 & 1502 & 34.215 & $\mathrm{C}_{15} \mathrm{H}_{24}$ & 204 \\
\hline 41. & Germacrene B & 0.0333 & 1550 & 36.215 & $\mathrm{C}_{15} \mathrm{H}_{24}$ & 204 \\
\hline 42. & Spathulenol & 0.0660 & 1569 & 36.972 & $\mathrm{C}_{15} \mathrm{H}_{24} \mathrm{O}$ & 220 \\
\hline 43. & Cadin-4-en-10-ol & 0.0242 & 1648 & 39.996 & $\mathrm{C}_{15} \mathrm{H}_{26} \mathrm{O}$ & 222 \\
\hline 44. & Cis Thijopsenic acid & 0.0460 & 1819 & 46.136 & $\mathrm{C}_{15} \mathrm{H}_{22} \mathrm{O}_{2}$ & 234 \\
\hline 45. & Torulosol & 0.0912 & 1851 & 47.380 & $\mathrm{C}_{20} \mathrm{H}_{34} \mathrm{O}_{2}$ & 306 \\
\hline
\end{tabular}

Table 5c. Composition of essential oil compounds at 1200-1400m altitude in immature stage.

\begin{tabular}{|c|c|c|c|c|c|c|}
\hline $\begin{array}{l}\text { 1246m } \\
\text { S.No. }\end{array}$ & Compound name & Area \% & $\overline{\mathbf{R I}}$ & R. Time & $\begin{array}{l}\text { Molecular } \\
\text { formula }\end{array}$ & $\begin{array}{l}\text { Molecular } \\
\text { weight }\end{array}$ \\
\hline 1. & a-Thujene & 0.2244 & 928 & 7.437 & $\mathrm{C}_{10} \mathrm{H}_{16}$ & 136 \\
\hline 2. & $\alpha$-Pinene & 0.9593 & 935 & 7.710 & $\mathrm{C}_{10} \mathrm{H}_{16}$ & 136 \\
\hline 3. & Sabinene & 1.5533 & 977 & 9.263 & $\mathrm{C}_{10} \mathrm{H}_{16}$ & 136 \\
\hline 4. & $\beta$-Pinene & 0.5822 & 979 & 9.370 & $\mathrm{C}_{10} \mathrm{H}_{16}$ & 136 \\
\hline 5. & Myrcene & 1.6852 & 996 & 10.000 & $\mathrm{C}_{10} \mathrm{H}_{16}$ & 136 \\
\hline 6. & Octanal & 0.2687 & 1010 & 10.587 & $\mathrm{C}_{8} \mathrm{H}_{16} \mathrm{O}$ & 128 \\
\hline 7. & Limonene & 83.6993 & 1057 & 12.667 & $\mathrm{C}_{10} \mathrm{H}_{16}$ & 136 \\
\hline 8. & $\beta$-Ocimene & 0.1582 & 1060 & 12.800 & $\mathrm{C}_{10} \mathrm{H}_{16}$ & 136 \\
\hline 9. & Y-terpinene & 6.4489 & 1074 & 13.400 & $\mathrm{C}_{10} \mathrm{H}_{16}$ & 136 \\
\hline 10. & 1-Nonanol & 0.0450 & 1082 & 13.743 & $\mathrm{C}_{8} \mathrm{H}_{18} \mathrm{O}$ & 130 \\
\hline 11. & Terpinolene & 0.3123 & 1093 & 14.263 & $\mathrm{C}_{10} \mathrm{H}_{16}$ & 136 \\
\hline 12. & Linalool & 2.0481 & 1113 & 15.160 & $\mathrm{C}_{10} \mathrm{H}_{18} \mathrm{O}$ & 154 \\
\hline 13. & $\beta$-Terpineol & 0.0176 & 1131 & 15.983 & $\mathrm{C}_{10} \mathrm{H}_{18} \mathrm{O}$ & 154 \\
\hline 14. & Cosmene & 0.0095 & 1145 & 16.647 & $\mathrm{C}_{10} \mathrm{H}_{14}$ & 134 \\
\hline 15. & Citronellal & 0.0455 & 1157 & 17.220 & $\mathrm{C}_{10} \mathrm{H}_{18} \mathrm{O}$ & 154 \\
\hline 16. & 4-Terpineol & 0.4371 & 1186 & 18.577 & $\mathrm{C}_{10} \mathrm{H}_{18} \mathrm{O}$ & 154 \\
\hline 17. & $\alpha$-terpineol & 0.2537 & 1201 & 19.270 & $\mathrm{C}_{10} \mathrm{H}_{18} \mathrm{O}$ & 154 \\
\hline 18. & Decanal & 0.1306 & 1211 & 19.740 & $\mathrm{C}_{10} \mathrm{H}_{20} \mathrm{O}$ & 156 \\
\hline 19. & p-cymene & 0.0935 & 1234 & 20.757 & $\mathrm{C}_{11} \mathrm{H}_{16} \mathrm{O}$ & 164 \\
\hline 20. & Neral & 0.0266 & 1242 & 21.153 & $\mathrm{C}_{10} \mathrm{H}_{16} \mathrm{O}$ & 152 \\
\hline 21. & Cis-citral & 0.0471 & 1272 & 22.527 & $\mathrm{C}_{10} \mathrm{H}_{16} \mathrm{O}$ & 152 \\
\hline 22. & Perillaldehyde & 0.0303 & 1278 & 22.780 & $\mathrm{C}_{10} \mathrm{H}_{16} \mathrm{O}$ & 152 \\
\hline 23. & Thymol & 0.4106 & 1302 & 23.877 & $\mathrm{C}_{10} \mathrm{H}_{14} \mathrm{O}$ & 150 \\
\hline 24. & $\alpha$-Terpinolene & 0.0457 & 1337 & 25.390 & $\mathrm{C}_{15} \mathrm{H}_{24}$ & 204 \\
\hline 25. & $\beta$-Elemene & 0.0156 & 1390 & 27.733 & $\mathrm{C}_{15} \mathrm{H}_{24}$ & 204 \\
\hline 26. & Do-decanal & 0.0169 & 1414 & 28.723 & $\mathrm{C}_{12} \mathrm{H}_{24} \mathrm{O}$ & 184 \\
\hline 27. & Germacrene B & 0.0076 & 1430 & 29.403 & $\mathrm{C}_{15} \mathrm{H}_{24}$ & 204 \\
\hline 28. & $\beta$-Farnescene & 0.0445 & 1455 & 30.457 & $\mathrm{C}_{15} \mathrm{H}_{24}$ & 204 \\
\hline 29. & Germacrene-D & 0.0650 & 1480 & 31.500 & $\mathrm{C}_{15} \mathrm{H}_{24}$ & 204 \\
\hline 30. & Y-elemene & 0.0064 & 1494 & 32.077 & $\mathrm{C}_{15} \mathrm{H}_{24}$ & 204 \\
\hline 31. & a -bergamotene & 0.0530 & 1506 & 32.583 & $\mathrm{C}_{15} \mathrm{H}_{24}$ & 204 \\
\hline 32. & Bicyclogermacrene & 0.0778 & 1557 & 34.577 & $\mathrm{C}_{15} \mathrm{H}_{24}$ & 204 \\
\hline 33. & $\beta$-eudesmol & 0.0112 & 1656 & 38.357 & $\mathrm{C}_{15} \mathrm{H}_{26} \mathrm{O}$ & 222 \\
\hline 34. & $\beta$-sinensal & 0.0496 & 1697 & 39.890 & $\mathrm{C}_{15} \mathrm{H}_{22} \mathrm{O}$ & 218 \\
\hline 35. & $\alpha$-bergamotol & 0.1197 & 1754 & 41.930 & $\mathrm{C}_{15} \mathrm{H}_{22} \mathrm{O}$ & 218 \\
\hline
\end{tabular}


Pradhan, A. et al. / J. Appl. \& Nat. Sci. 11(1): 168-181 (2019)

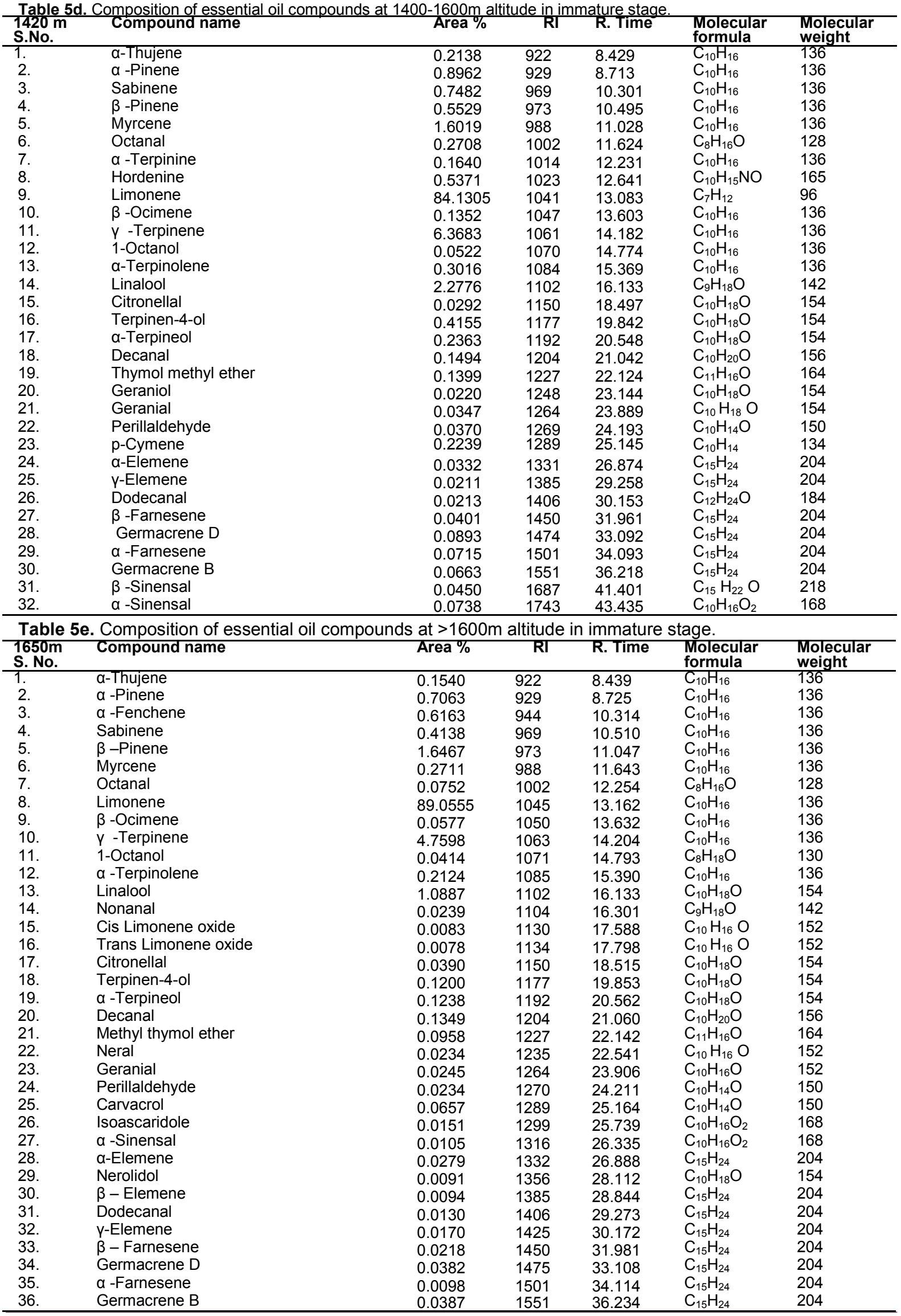


73.09 , 88.46), however, it was highest at $>1600 \mathrm{~m}$ lowest at $1400-1600 \mathrm{~m}$. Likewise, $\mathrm{Y}$-Terpinene was found to be $4.82 \%$ being maximumin the samples from $1200-1400 \mathrm{~m}$ altitude while $3.99 \%$ at $>1600 \mathrm{~m}$ was the lowest content. In addition myrcene was found maximum (1.64\%) at $800-1000 \mathrm{~m}$ altitude followed by $1.61 \%$ at $>1600 \mathrm{~m}$ altitude. While at $1400-1600 m$ only $1.44 \%$ myrcenewas recorded lowest. Further, linalool $(9.13 \%)$ was accorded maximum at $1400-1600 \mathrm{~m}$ altitude and minimum of $1.46 \%$ at $800-1000 \mathrm{~m}$ altitudeThe result were in corroboration with the finding of Javed et al., 2014. They had mentioned that limoneneas the highest which was in the range of $58.50 \%$ (mandarin essential oil) to $89.84 \%$ (grape fruit essential oil).

Likewise in immature stage,40,45, 35, 32 and 36 compounds were found in $800,1000-1200,1200-$ $1400,1400-1600$ and $>1600 \mathrm{~m}$ altitudes respectively (Table 5a,5b,5c,5d). Amongst this, $87.93 \%$, $88.58 \%, 83.70 \%, 84.13 \%, 89.06 \%$ of limonene were present in 800,1000-1200, 1200-1400, 14001600 and $>1600 \mathrm{~m}$ altitudes. It showed $>1600 \mathrm{~m}$ altitude with highest content of limonene. Thereafter, highest $\mathrm{Y}$-Terpinene was found in 1200$1400 \mathrm{~m}(6.45 \%)$ followed by $1400-1600 \mathrm{~m}(6.37 \%)$ and of the least was found at $1000-1200 \mathrm{~m}$ $(1.42 \%)$.Myrcene content was lowest at $>1600 \mathrm{~m}$ $(0.27 \%)$ and highest at $1200-1400 \mathrm{~m} 1.68 \%$. Linalool was found in the range of $0.92 \%$ (1000$1200 \mathrm{~m})$ to $2.28 \%(1400-1600 \mathrm{~m})$. Whereas, $\mathrm{a}-$ Pinene on was found highest at at $1200-1400 \mathrm{~m}$ altitude $(0.96 \%)$ with highest value and that of $0.38 \%$ at $1400-1600 \mathrm{~m}$ as the lowest content. Earlier worker (Wu et al., 2014) reported chemical content of oil from Taiwan in limonene upto $57.71 \%, 86.05 \%$ in lemon and orange oil and $\alpha-$ Pineneup to $2.27 \%, 0.58 \%$, Myrcene upto $1.44 \%$, $2.22 \%$. d-limoneneof Moro blood orange oil was reported upto93.32\%and in Mayer lemon it was $75.50 \%$, while Interdonat lemon was noted with $66.58 \%$. The other main components of oils were determined to be $\alpha$-pinene, sabinene, $\beta$ pinene, $\beta$-myrcene, linalool, m-cymene and 4terpineol in addition to d-limonene.

Kamal et al. (2011) performed research in C. reticulata, $C$ sinensis and C.paradisii in fresh, ambient and air dried condition in Pakistan. It revealed that C.reticulataconstituted 27,27 and 16 compounds, representing upto99.98, 99.50 and $97.25 \%$ of the total oil. The major compound werelimonene (69.9, 64.1and $71.1 \%$ ), followed by $\beta$-myrcene (3.27, 4.05 and $4.02 \%$ ) and decanal $(2.33,7.71$ and $5.80 \%$ ) in fresh, ambient-driedand oven-dried peel oil.

\section{Conclusion}

Quantitative analysis of the peel of Citrus showed increase in altitude increase in all the parameters like percent yield on dry basis, percent yield on wet basis, equivalent weight, methoxyl content, degree of esterification, moisture content, ash percent, alkalinity as carbonate, carbonate free ash. In FTIR analysis, highest altitude $(>1600 \mathrm{~m})$ showed $\mathrm{C}-\mathrm{H}$ stretch and that of $\mathrm{O}-\mathrm{H}$ bond was found at $800 \mathrm{~m}$ altitude respectively Moreover mature stage showed $39,33,51,56,43$ at $800 \mathrm{~m}$, $1000-1200 \mathrm{~m}, 1200-1400 \mathrm{~m}, 1400-1600 \mathrm{~m}$ and $>1600 \mathrm{~m}$ altitudes. Limonene content showed $88.46 \%$ at $>1600 \mathrm{~m}$ altitude. Likewise $\mathrm{y}$-Terpinene was found to be $4.82 \%$ being maximum in the samples from $1200-1400 \mathrm{~m}$ altitude while $3.99 \%$ at $>1600 \mathrm{~m}$ was the lowest content. In addition, myrcene was found maximum at $800-1000 \mathrm{~m}$ altitude $(1.64 \%)$ while $1400-1600 \mathrm{~m}(1.44 \%)$ was recorded with lowest value. Immature stage on the other hand was found with $89.06 \%$ at $>1600 \mathrm{~m}$ altitude. Likewise $\mathrm{Y}$-Terpinene was found in 1200-1400m $(6.45 \%)$ followed by $1400-1600 \mathrm{~m}(6.37 \%)$ and of the least was found at $1000-1200 \mathrm{~m}$ $(1.42 \%)$ ).Myrcene content was lowest at $>1600 \mathrm{~m}$ $(0.27 \%)$ and highest at $1200-1400 \mathrm{~m} 1.68 \%$. The present research showed significant amount of component present and in which limonene was found to be the highest followed by $\mathrm{y}$-Terpinene, myrcene. Hence the essential oil has significance as a natural source of antimicrobials for therapeutic purposes. The identification of compound may be helpful in the study as well as medicinal properties.

\section{ACKNOWLEDGEMENTS}

Authors acknowledge the farmers at five different altitudes in four districts of Sikkim who had provided the fruits. Acknowledgement is also given to the Department of Horticulture, Sikkim University for providing needed facilities for this study.

\section{REFERENCES}

1. Adams, R.P. (2001). Identification of essential oil components by gas chromatography/quadrupole mass spectrometry. Scientific Research. An Academic Publisher, Allured Publishing Corporation, Carol Stream, p 455.

2. Bagde, P.P., Dhenge, S., and Bhivgade, S. (2017).Extraction of pectin from orange peel and lemon peel. International Journal of Engineering Technology Science and Research, 4 (3): 1-7

3. Brouns, F., Theuwissen, E., Adam, A., Bell, M., Berger, A., and Mensink, R.P.(2012). Cholesterol -lowering properties of different pectin types in mildly hyper-cholesterolemic men and women. European Journal of Clinical Nutrition, 66(5): 591-900.

4. Chin, L.S., Chin, N.L., andYusof, Y.A. (2014). Extraction and characterization of pectin from passion fruit peels. Agriculture and Agricultural Science Procedia, 2: 231-236.

5. Devi, W.E., Shukla, R.N., Abraham, A., Jarpula, S., and Kaushik, U. (2014). Optimized Extraction condition and characterization of pectin from orange Peel, IJREAT, 2: 1-9.

6. Fakayode, O.A., and Abobi, K. E.(2018).Optimization 
of oil and pectin extraction from orange (Citrus sinensis) peels: a response surface approach. Journal of Analytical Science and Technology, 9: 20

7. Ismail, A., Lukman, S., Ojo, S. O., Bolorunduro, K. A., Adeosun, O. O., andOke, I. A. (2016). Solutions of selected pseudo loop equations in water distribution network using microsoft excel solver. Ife Journal of Science, 18 (2): 371-387

8. Javed, S., Javaid, A., Nawaz, S., Saeed, M.K., Mahmood, Z., Siddiqui, S.Z and Ahmad, R.2014.Phytochemistry, GC-MS Analysis, Antioxidant and Antimicrobial Potential of Essential Oil From Five Citrus Species. Journal of Agricultural Sciences, 6 (3): 201-208

9. Joslyn, M.N. (1980). Methods of food analysis, physical chemical and instrumentation method of analysis. Academic Press, New York, 5, 67-70.

10.Joye, D.D. and G.A. Luzio, 2000. Process for Selective Extraction of Pectin from Plant Material by Differential $\mathrm{pH}$. Journal of Carbohydrate Polymer, 43(4): 337-342.

11.Kamal, G. M., Anwar, F., Hussain, A. I., Sarri, N., and Ashraf, M. Y. (2011). Yield and chemical composition of Citrus essential oils as affected by drying pre treatment of peels. International Food Research Journal, 18(4): 1275-1282

12.Kanmani, P., Dhivya, E., Aravind, J., and Kumaresan, K. (2014). Extraction and Analysis of Pectin from Citrus Peels: Augmenting the yield from Citrus limon using statistical experimental design. Iranica Journal of Energy and Environment, 5 (3):303-312

13.Khule, R.N., Nitin, B.M., Dipak, S.S., Manisha, M.R., and Sanjay, R.C.(2012). Extraction of pectin from citrus fruit peel and use as natural binder in paracetamol tablet. Scholars Research Library, 4(2):558-564.

14.Krishnamurthi, C.R., and Giri, K.V. (2003). Preparation, purification and composition of pectin from Indian fruits and vegetables. Brazilian Archives of Biology and Technology, 44: 476-483.

15.Kulkarni, G.T., Gowthmarajan, K., Rao, B., and Suresh, B.(2006). Evaluation of Binding Properties of Blantago ovate and Trigonella foenum graecum mu- cilages. Indian Drugs,39(8), 422-425.

16.Norziah, M.H., Fang, E.O.,andKarim, A.A.(2000). Extraction and characterization of pectin from pomelo fruit peels. In P.A. Williams (Ed.), Gums and stabilisers for the food industry, Cambridge, UK: The Royal Society of Chemistry, 10: 26-36.

17.Owens, H.S., Mc Cready, R.M., Shepherd, A.D., Schultz, S.H., Pippen, E.L., Swenson, H.A.,.Miers, J.C., Erlandsen, R.F., andMaclay, W.D. (1952). Methods used at western regional research laboratory for extraction and analysis of pectic materials, AIC-340, Western Regional Research Laboratory, Albany California.

18.Pagan, J., Ibarz, A., Llorca, M., and Paga, A. (2001). Extraction and characterization of pectin from for the extraction of pectin from stored peach pomace. Food Research International, 34: 605-612

19.Ranganna, (1986). Handbook of analysis and quality control for fruit and vegetable products. Tata McGraw -Hill Education Private Limited. New Delhi, p 33.

20.Schultz. (1976). Methods in Carbohydrate Chemistry. In T. Schultz, Methods in Carbohydrate Chemistry, New York, Academic Press, pp 189.

21.Tobias, N.E., Eke, N.V., Okechukwu, R.I., Nwoguikpe, R.N.,andDuru, C.M. (2011). Waste to health: Industrial raw materials. Potential of peels of Nigerian sweet orange (Citrus sinensis). African Journal of Biotechnology, 10(33): 6257-6264.

22. Tiwari, A.K., Saha, S.N., Yadav, V.P., Uadhyay, U.K., Katiyar, D., and Mishra, T. (2017).Extraction and characterization of pectin from orange peels. International Journal of Biotechnology and Biochemistry, 13: 39-47.

23.Wu, P.S., Kuo, Y.T., Chen, S.M., Li, Y ., and Lou, S. B.(2014). Gas Chromatography-Mass Spectrometry Analysis of Photosensitive Characteristics in Citrus and Herb Essential Oils. Journal of Chromatrography Separation Techniques, 6(1): 1-9.

24.Yadav, S.D, Bankar, N.S., Waghmare, N.N., andShete, D.C. (2017). Extraction and characterization of pectin from sweet lime. 4th International Conference on Multidisciplinary Research and Practice, pp. 58-63. 